\title{
Linear-Time Compression of Bounded-Genus Graphs into Information-Theoretically Optimal Number of Bits*
}

\author{
Hsueh-I Lu ${ }^{\dagger}$ \\ January 11, 2014
}

\begin{abstract}
A compression scheme $A$ for a class $\mathbb{G}$ of graphs consists of an encoding algorithm Encode $_{A}$ that computes a binary string $\operatorname{Code}_{A}(G)$ for any given graph $G$ in $\mathbb{G}$ and a decoding algorithm Decode ${ }_{A}$ that recovers $G$ from $\operatorname{Code}_{A}(G)$. A compression scheme $A$ for $\mathbb{G}$ is optimal if both Encode $_{A}$ and Decode $_{A}$ run in linear time and the number of bits of $\operatorname{Code}_{A}(G)$ for any $n$-node graph $G$ in $\mathbb{G}$ is information-theoretically optimal to within lower-order terms. Trees and plane triangulations were the only known nontrivial graph classes that admit optimal compression schemes. Based upon Goodrich's separator decomposition for planar graphs and Djidjev and Venkatesan's planarizers for bounded-genus graphs, we give an optimal compression scheme for any hereditary (i.e., closed under taking subgraphs) class $\mathbb{G}$ under the premise that any $n$-node graph of $\mathbb{G}$ to be encoded comes with a genus- $o\left(\frac{n}{\log ^{2} n}\right)$ embedding. By Mohar's lineartime algorithm that embeds a bounded-genus graph on a genus- $O(1)$ surface, our result implies that any hereditary class of genus- $O(1)$ graphs admits an optimal compression scheme. For instance, our result yields the first-known optimal compression schemes for planar graphs, plane graphs, graphs embedded on genus- 1 surfaces, graphs with genus 2 or less, 3-colorable directed plane graphs, 4-outerplanar graphs, and forests with degree at most 5 . For non-hereditary graph classes, we also give a methodology for obtaining optimal compression schemes. From this methodology, we give the first known optimal compression schemes for triangulations of genus$O(1)$ surfaces and floorplans.
\end{abstract}

*Accepted to SIAM Journal on Computing. A preliminary version appeared in SODA [65].

†Department of Computer Science and Information Engineering, National Taiwan University. Address: 1 Roosevelt Road, Section 4, Taipei 106, Taiwan, ROC. Web: www.csie.ntu.edu.tw/ hil/. Email: hil@csie.ntu.edu.tw. Research supported in part by NSC grant 101-2221-E-002-062-MY3. The author also holds joint appointments from the Graduate Institute of Networking and Multimedia and the Graduate Institute of Biomedical Electronics and Bioinformatics, National Taiwan University. 


\section{Introduction}

Compact representation of graphs are fundamentally important and useful in many applications, including representing the meshes in finite-element analysis, terrain models of GIS, and 3D models of graphics [80, 82, 81, 92, 85, 64, 89, 48], VLSI design [84, 56], designing compact routing tables of computer networks [94, 37, 66, 77, 35, 95, 1, 16, 3, 36], and compressing the link structure of the Internet [15, 2, 88, 7, 5, 21]. Let $\mathbb{G}$ be a class of graphs. Let $\operatorname{num}(\mathbb{G}, n)$ denote the number of distinct $n$-node graphs in $\mathbb{G}$. The informationtheoretically optimal number of bits to encode an $n$-node graph in $\mathbb{G}$ is $\lceil\log n u m(\mathbb{G}, n)\rceil 1$ For instance, if $\mathbb{G}$ is the class of rooted trees, then $\operatorname{num}(\mathbb{G}, n) \approx \frac{2^{2 n}}{n^{3 / 2}}$ and $\log n u m(\mathbb{G}, n)=$ $2 n-O(\log n)$; if $\mathbb{G}$ is the class of plane triangulations, then $\log n u m(\mathbb{G}, n)=\log \frac{256}{27} n+$ $o(n) \approx 3.2451 n+o(n)$ [97]. A compression scheme $A$ for $\mathbb{G}$ consists of an encoding algorithm Encode $_{A}$ that computes a binary string $\operatorname{Code}_{A}(G)$ for any given graph $G$ in $\mathbb{G}$ and a decoding algorithm Decode $_{A}$ that recovers graph $G$ from $\operatorname{Code}_{A}(G)$. A compression scheme $A$ for a graph class $\mathbb{G}$ with $\log n u m(\mathbb{G}, n)=O(n)$ is optimal if the following three conditions hold.

Condition C1: The running time of algorithm $\operatorname{Encode}_{A}(G)$ is linear in the size of $G$.

Condition C2: The running time of algorithm $\operatorname{Decode}_{A}\left(\operatorname{Code}_{A}(G)\right)$ is linear in the bit count of $\operatorname{Code}_{A}(G)$.

Condition C3: For all positive constants $\beta$ with $\log n u m(\mathbb{G}, n) \leq \beta n+o(n)$, the bit count of Code $_{A}(G)$ for an $n$-node graph $G$ in $\mathbb{G}$ is no more than $\beta n+o(n)$.

Condition C3 basically says that the bit count of $\operatorname{Code}_{A}(G)$ is information-theoretically optimal to within lower-order terms. Although there has been considerable work on compression schemes, trees (see e.g., [72, 50, 67, 11]) and plane triangulations [79] were the only known nontrivial graph classes that admit optimal compression schemes. A graph class is hereditary if it is closed under taking subgraphs. Below is the main result of the paper.

Theorem 1.1. Any hereditary class $\mathbb{G}$ of graphs with $\log n u m(\mathbb{G}, n)=O(n)$ admits an optimal compression scheme, as long as each input n-node graph in $\mathbb{G}$ to be encoded comes with a genus$o\left(\frac{n}{\log ^{2} n}\right)$ embedding.

By Theorem 1.1 and Mohar's linear-time genus- $O(1)$ embedding algorithm for genus$O(1)$ graphs [70, 54] (see Lemma 2.5), any hereditary class of genus- $O(1)$ graphs admits an optimal compression scheme. For instance, our result yields the first-known optimal compression schemes for planar graphs, plane graphs, graphs embedded on genus-1 surfaces, graphs with genus 2 or less, 3-colorable directed plane graphs, 4-outerplanar graphs, and forests with degree at most 5 . For non-hereditary graph classes, we also give an extension (see Corollary 5.1) of Theorem 1.1. As summarized in the following theorem, we show two classes of genus- $O(1)$ graphs whose optimal compression schemes

\footnotetext{
${ }^{1}$ All logarithms throughout the paper are to the base of two.
} 
are obtainable via this extension, where the class of floorplans is defined in related work below.

Theorem 1.2. The following classes of graphs admit optimal compression schemes:

1. Triangulations of a genus- $g$ surface for any integral constant $g$.

2. Floorplans.

Technical overview The kernel of the proof of Theorem 1.1 is a linear-time disjoint partition $G_{0}, \ldots, G_{p}$ of an $n$-node graph $G$ embedded on a genus- $O\left(\frac{n}{\log ^{2} n}\right)$ surface.2 Let poly $(n)$ denote $O\left(n^{O(1)}\right)$. Based upon Goodrich's separator decomposition of planar graphs [40] and Djidjev and Venkatesan's planarizer [26], partition $G_{0}, \ldots, G_{p}$ satisfies the following conditions, where $n_{i}$ is the number of nodes of $G_{i}$ and $d_{i}$ is the number of times that the nodes of $G_{i}$ are duplicated in some $G_{j}$ with $j \neq i \cdot \sqrt{3}$ (a) $n_{0}=o\left(\frac{n}{\log n}\right)$, (b) $n_{i}=\operatorname{poly}(\log n)$ holds for each $i=1,2, \ldots, p$, (c) $\sum_{i=1}^{p} d_{i}=o\left(\frac{n}{\log n}\right)$, and (d) $\sum_{i=0}^{p} n_{i}=n+o\left(\frac{n}{\log n}\right)$. By Condition (a), $G_{0}$ can be encoded in $o(n)$ bits. By Conditions (b) and (c), the information required to recover $G$ from $G_{0}, G_{1}, \ldots, G_{p}$ can be encoded into $o(n)$ bits (see Lemma4.1). By Condition (d), we have $\log \operatorname{num}(\mathbb{G}, n) \leq o(n)+\sum_{i=1}^{p} \log n u m\left(\mathbb{G}, n_{i}\right)$. Therefore, the disjoint partition reduces the problem of encoding an $n$-node graph in $\mathbb{G}$ to the problem of encoding a poly $(\log n)$-node graph in $\mathbb{G}$. Applying such a reduction for one more level, it remains to encode a poly $(\log \log n)$-node graph in $\mathbb{G}$ into an information-theoretically optimal number of bits, which can be resolved by the standard technique (see, e.g., [47, 72, 78]) of precomputation tables (see Lemma 2.3).

Related work The compression scheme of Turán [96] encodes an $n$-node plane graph that may have self-loops into $12 n$ bits 4 Keeler and Westbrook [55] improved this bit count to $10.74 n$. They also gave compression schemes for several families of plane graphs. In particular, they used $4.62 n$ bits for plane triangulation, and $9 n$ bits for connected plane graphs free of self-loops and degree-one nodes. For plane triangulations, He et al. [46] improved the bit count to $4 n$. For triconnected plane graphs, He et al. [46] also improved the bit count to at most $8.585 n$ bits. This bit count was later reduced to at most $\frac{9 \log _{2} 3}{2} n \approx$ $7.134 n$ by Chuang et al. [20]. For any given $n$-node graph $G$ embedded on a genus- $g$ surface, Deo and Litow [25] showed an an $O(n g)$-bit encoding for $G$. These compression schemes all take linear time for encoding and decoding, but Condition $\mathrm{C} 3$ does not hold for them. The compression schemes of He et al. [47] (respectively, Blelloch et al. [14]) for planar graphs, plane graphs, and plane triangulations (respectively, separable graphs) satisfies Condition $\mathrm{C} 3$, but their encoding algorithms require $\Omega(n \log n)$ time on $n$-node graphs.

\footnotetext{
${ }^{2}$ Precisely, the disjoint partition $G_{0}, \ldots, G_{p}$ of the edges of the embedded graph $G$ in the proof of Theorem 1.1 is $G\left[V_{0}\right], G\left(V_{1}\right), \ldots, G\left(V_{p}\right)$, where $\left[V_{0}, \ldots, V_{p}\right]$ is both (i) a 1-separation $\mathbb{S}_{1}$ of an arbitrary triangulation $\Delta$ of $G$ and (ii) a refinement of the 0 -separation $\mathbb{S}_{0}=[\varnothing, \operatorname{Node}(\Delta)]$ of $\Delta$.

${ }^{3}$ As a matter of fact, in our construction, all duplicated nodes of $G_{i}$ with $i \geq 1$ belong to $G_{0}$.

${ }^{4}$ For brevity, we omit all lower-order terms of bit counts in our discussion of related work.
} 


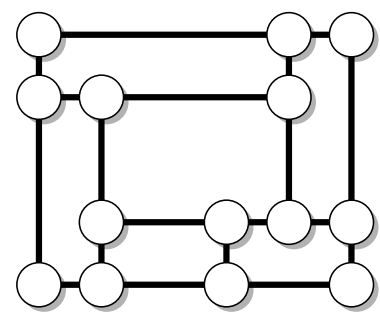

(a)

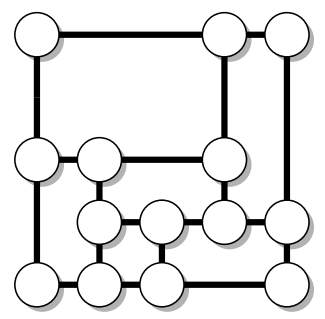

(b)

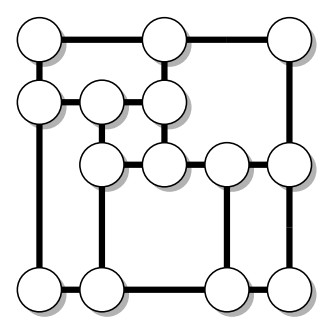

(c)

Figure 1: Three floorplans with 14 nodes, 6 internal faces, and 19 edges. Floorplans (a) and (b) are equivalent, floorplans (b) and (c) are not equivalent.

Floorplanning is a fundamental issue in circuit layout [106, 43, 69, 62, 51, 108, 32, 8, 17, 58, 24, 57, 91, 68, 84, 4]. Motivated by VLSI physical design, various representations of floorplans were proposed [110, 109, 33]. Designing a floorplan to meet a certain criterion is NP-complete in general [87, 44, 100], so heuristic techniques such as simulated annealing [102, 101, 17] are practically useful. The length of the encoding affects the size of the search space. A floorplan, which is also known as rectangular drawing, is a division of a rectangle into rectangular faces using horizontal and vertical line segments. Two floorplans are equivalent if they have the same adjacency relations and relative positions among the nodes. For instance, Figure 1 shows three floorplans: Floorplans (a) and (b) are equivalent. Floorplans (b) and (c) are not equivalent. Let $G$ be the input $n$-node floorplan. Under the conventional assumption that each node of $G$, other than the four corner nodes, has exactly three neighbors (see, e.g., [45, 107]), one can verify that $G$ has $0.5 n$ faces and 1.5n - 2 edges. Yamanaka and Nakano [103] showed how to encode $G$ into $2.5 n$ bits. Chuang [19] reduced the bit count to 2.293n. Takahashi et al. [90] further reduced bit count to $2 n$. All these compression schemes for floorplans satisfy Conditions C1 1 and $\mathrm{C} 2$, but not Condition C3, Takahashi et al. [90] also showed that the number of distinct $n$ node floorplans is no more than $3.375^{n+o(n)} \approx 2^{1.755 n+o(n)}$. Therefore, our Theorem 1.2(2) encodes an $n$-node floorplan into at most $1.755 n$ bits.

For applications that require query support, Jacobson [50] gave a $\Theta(n)$-bit encoding for a connected and simple planar graph $G$ that supports traversal in $\Theta(\log n)$ time per node visited. Munro and Raman [71] improved this result and gave schemes to encode binary trees, rooted ordered trees, and planar graphs. For a general $n$-node $m$-edge planar graph $G$, they used $2 m+8 n$ bits while supporting adjacency and degree queries in $O(1)$ time. Chuang et al. [20] reduced this bit count to $2 m+\left(5+\frac{1}{k}\right) n$ for any constant $k>0$ with the same query support. The bit count can be further reduced if only $O(1)$-time adjacency queries are supported, or if $G$ is simple, triconnected or triangulated [20]. Chiang et al. [18] reduced the number of bits to $2 m+2 n$. Yamanaka and Nakano [105] showed a $6 n$-bit encoding for plane triangulations with query support. The succinct encodings of Blandford et al. [13] and Blelloch et al. [14] for separable graphs support queries. Yamanaka et al. [104] also gave a compression scheme for floorplans with query support. For labeled planar graphs, Itai and Rodeh [49] gave an encoding 
of $\frac{3}{2} n \log n$ bits. For unlabeled general graphs, Naor [74] gave an encoding of $\frac{1}{2} n^{2}$ bits. For certain graph families, Kannan et al. [52] gave schemes that encode each node with $O(\log n)$ bits and support $O(\log n)$-time testing of adjacency between two nodes. Galperin and Wigderson [34] and Papadimitriou and Yannakakis [75] investigated complexity issues arising from encoding a graph by a small circuit that computes its adjacency matrix. Related work on various versions of succinct graph representations can be found in $[73,6,31,42,38,76,83,30,29,28,9,53]$ and the references therein.

Outline The rest of the paper is organized as follows. Section 2 gives the preliminaries. Section 3 shows our algorithm for computing graph separations. Section 4 gives our optimal compression scheme for hereditary graph classes. Section 5 shows a methodology for obtaining optimal compression schemes for non-hereditary graph classes and applies this methodology on triangulations of genus- $O(1)$ graphs and floorplans. Section 6 concludes the paper with a couple of open questions.

\section{Preliminaries}

\subsection{Segmentation prefix}

Let $\|X\|$ denote the number of bits of binary string $X$. A binary string $X_{0}$ is a segmentation prefix of binary strings $X_{1}, \ldots, X_{d}$ if (a) it takes $O\left(\sum_{i=1}^{d}\left\|X_{i}\right\|\right)$ time to compute $X_{0}$ from $X_{1}, \ldots, X_{d}$ and (b) given the concatenation of $X_{0}, X_{1}, \ldots, X_{d}$, it takes $O\left(\sum_{i=0}^{d}\left\|X_{i}\right\|\right)$ time to recover all $X_{i}$ with $1 \leq i \leq d$.

Lemma 2.1 (See, e.g., [10, 27]). Any binary strings $X_{1}, \ldots, X_{d}$ with $d=O(1)$ have a segmentation prefix with $O\left(\log \sum_{i=1}^{d}\left\|X_{i}\right\|\right)$ bits.

Lemma 2.2. Any binary strings $X_{1}, \ldots, X_{d}$ have an $O(\min \{m, d \log m\})$-bit segmentation prefix, where $m=\left\|X_{1}\right\|+\cdots+\left\|X_{d}\right\|$.

Proof. Let $X$ be the concatenation of $X_{1}, \ldots, X_{d}$. If $m \leq d \log m$, let $X^{\prime}$ be the $m$-bit binary string with exactly $d$ copies of 1-bits such that the $j$-th bit of $X^{\prime}$ is 1 if and only if $j=$ $\left\|X_{1}\right\|+\cdots+\left\|X_{i}\right\|$ holds for some $i=1, \ldots, d$. Otherwise, let $X^{\prime}$ store the $O(\log m)$-bit numbers $\left\|X_{1}\right\|+\cdots+\left\|X_{i}\right\|$ for all $i=1, \ldots, d$. Let $X_{0}^{\prime}$ be the segmentation prefix of $X^{\prime}$ and $X$ as ensured by Lemma 2.1. The concatenation of $X_{0}^{\prime}$ and $X^{\prime}$ is a segmentation prefix $X_{0}$ of $X_{1}, \ldots, X_{d}$ with $O(\min \{m, d \log m\})$ bits. The lemma is proved.

For the rest of the paper, let $X_{1} \circ \cdots \circ X_{d}$ denote the concatenation of $X_{0}, X_{1}, \ldots, X_{d}$, where $X_{0}$ is the segmentation prefix of $X_{1}, \ldots, X_{d}$ as ensured by Lemma 2.2. 


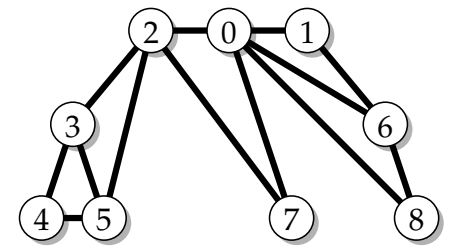

(a)

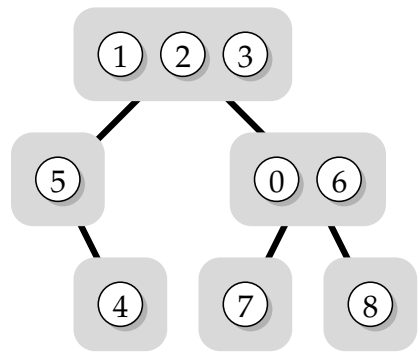

(b)

Figure 2: (a) A 9-node plane graph $G$. (b) A separator decomposition $\mathbb{T}$ of $G$.

\subsection{Precomputation table}

Unless clearly stated otherwise, all graphs throughout the paper are simple, i.e., having no multiple edges or self-loops. Let $|S|$ denote the cardinality of set $S$. Let $\operatorname{Node}(G)$ consist of the nodes in graph $G$ and let node $(G)=|\operatorname{Node}(G)|$. For any subset $V$ of $\operatorname{Node}(G)$, let $G[V]$ denote the subgraph of $G$ induced by $V$ and let $G \backslash V$ denote the subgraph of $G$ obtained by deleting $V$ and their incident edges. Two disjoint subsets $V$ and $V^{\prime}$ of $\operatorname{Node}(G)$ are adjacent in $G$ if there is an edge $\left(v, v^{\prime}\right)$ of $G$ with $v \in V$ and $v^{\prime} \in V^{\prime}$. For any subset $V$ of $\operatorname{Node}(G)$, let $\operatorname{Nbr}_{G}(V)$ consist of the nodes in $\operatorname{Node}(G) \backslash V$ that are adjacent to $V$ in $G$ and let $n b r_{G}(V)=\left|N b r_{G}(V)\right|$. A connected component of graph $G$ is a maximal subset $C$ of $\operatorname{Node}(G)$ such that $G[C]$ is connected.

Lemma 2.3. Let $\mathbb{G}$ be a graph class satisfying $\log n u m(\mathbb{G}, n)=O(n)$. Given positive integers $\ell$ and $n$ with $\ell=p o l y(\log \log n)$, it takes overall o( $n)$ time to compute (i) a labeling Label $(H)$ and a $\lceil\log$ num $(\mathbb{G}$, node $(H))\rceil$-bit binary string Optcode $(H)$ for each distinct graph $H \in \mathbb{G}$ with at most $\ell$ nodes and (ii) an o(n)-bit string Table $(\mathbb{G}, \ell)$ such that the following statements hold.

1. Given any graph $H \in \mathbb{G}$ with node $(H) \leq \ell$, it takes $O($ node $(H))$ time to obtain Optcode $(H)$ and Label $(H)$ from Table $(\mathbb{G}, \ell)$.

2. Given Optcode $(H)$ for any graph $H \in \mathbb{G}$ with node $(H) \leq \ell$, it takes $O($ node $(H))$ time to obtain $H$ and Label $(H)$ from Table $(\mathbb{G}, \ell)$.

Proof. Straightforward by $O(1)^{\text {poly }(\ell)}=o(n)$.

\subsection{Separator decomposition of planar graphs}

Sets $S_{1}, \ldots, S_{d}$ form a disjoint partition of set $S$ if $S_{1}, \ldots, S_{d}$ are pairwise disjoint and $S=$ $S_{1} \cup \cdots \cup S_{d}$. A subset $S$ of $\operatorname{Node}(G)$ is a separator of graph $G$ with respect to $S_{1}$ and $S_{2}$ if (1) $S, S_{1}$, and $S_{2}$ form a disjoint partition of $\operatorname{Node}(G),(2) S_{1}$ and $S_{2}$ are not adjacent in $G$, (3) $|S|=O\left(\operatorname{node}(G)^{1 / 2}\right)$, and (4) $\max \left\{\left|S_{1}\right|,\left|S_{2}\right|\right\} \leq \frac{2}{3} \cdot \operatorname{node}(G)$. A separator decomposition [12] of $G$ is a rooted binary tree $\mathbb{T}$ on a disjoint partition of $\operatorname{Node}(G)$ such that the following two statements hold, where "nodes" specify elements of Node $(G)$ and "vertices" specify elements of $\operatorname{Node}(\mathbb{T})$. Statement 1: Each leaf vertex of $\mathbb{T}$ consists of a single node of $G$. 


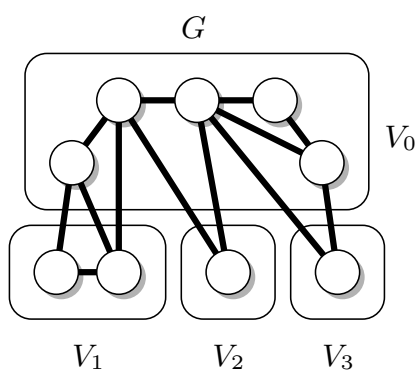

(a)

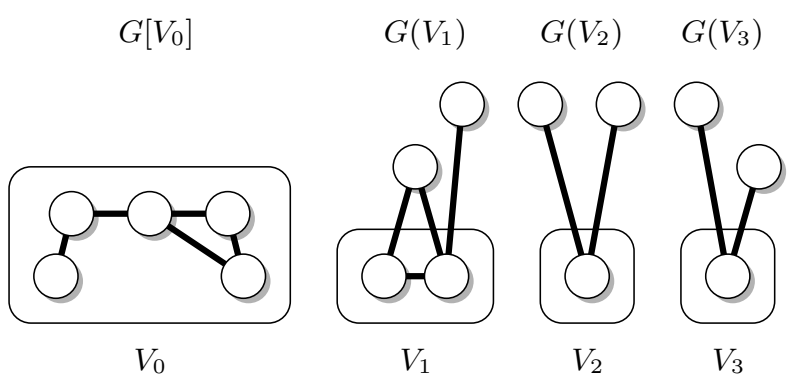

(b)

Figure 3: (a) A 9-node plane graph with a separation $\left[V_{0}, \ldots, V_{3}\right]$. (b) $G\left[V_{0}\right], G\left(V_{1}\right), G\left(V_{2}\right)$, and $G\left(V_{3}\right)$ form a disjoint partition of the edges of $G$.

Statement 2: Each internal vertex $S$ of $\mathbb{T}$ is a separator of $G[\operatorname{Offspring}(S)]$ with respect to $\operatorname{Offspring}\left(S_{1}\right)$ and $\operatorname{Offspring}\left(S_{2}\right)$, where $S_{1}$ and $S_{2}$ are the child vertices of $S$ in $\mathbb{T}$ and Offspring $(S)$ (respectively, Offspring $\left(S_{1}\right)$ and Offspring $\left(S_{2}\right)$ ) is the union of all the vertices in the subtree of $\mathbb{T}$ rooted at $S$ (respectively, $S_{1}$ and $S_{2}$ ). See Figure 2 for an illustration.

Lemma 2.4 (Goodrich [40]). It takes $O(n)$ time to compute a separator decomposition for any given n-node planar graph.

\subsection{Planarizers for non-planar graphs}

The genus of a graph $G$ is the smallest integer $g$ such that $G$ can be embedded on an orientable surface with $g$ handles without edge crossings [41]. For example, the genus of a planar graph is zero. By Euler's formula (see, e.g., [39]), an $n$-node genus- $O(n)$ graph has $O(n)$ edges. Determining the genus of a general graph is NP-complete [93], but Mohar [70] showed that it takes linear time to determine whether a graph is of genus $g$ for any $g=O(1)$. Mohar's algorithm is simplified by Kawarabayashi et al. [54].

Lemma 2.5 (Mohar et al. [70, 54]). It takes $O(n)$ time to compute a genus-O(1) embedding for any given $n$-node genus- $O(1)$ graph.

Gilbert et al. [39] gave an $O(n+g)$-time algorithm to compute an $O\left((g n)^{0.5}\right)$-node separator of an $n$-node genus- $g$ graph, generalizing Lipton and Tarjan's classic separator theorem for planar graphs [63]. Our result relies on the following planarization algorithm.

Lemma 2.6 (Djidjev and Venkatesan [26]). Given an n-node graph $G$ embedded on a genus- $g$ surface, it takes $O(n+g)$ time to compute a subset $V$ of Node $(G)$ with $|V|=O\left((g n)^{0.5}\right)$ such that $G \backslash V$ is planar.

\section{Separation and refinement}

We say that $\left[V_{0}, \ldots, V_{p}\right]$ with $p \geq 1$ is a separation of graph $G$ if the following properties hold. 


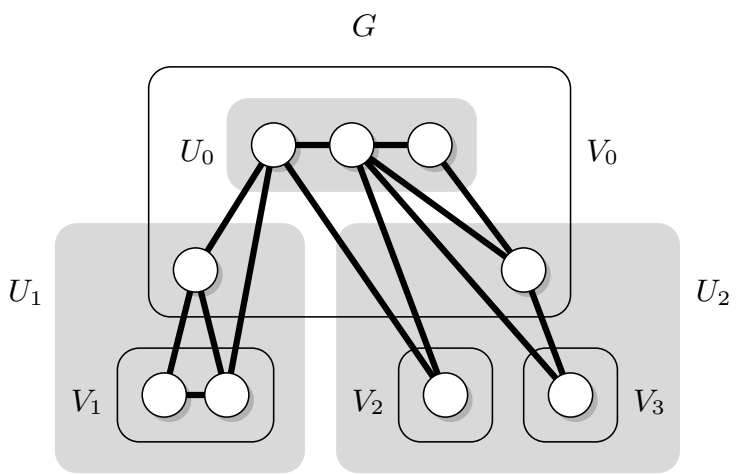

(a)

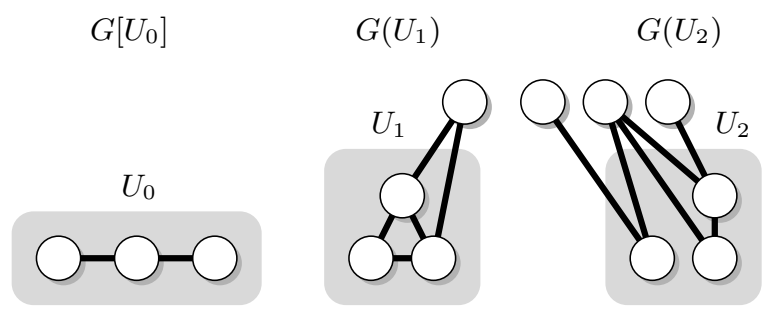

(b)

Figure 4: (a) Separation $\left[V_{0}, V_{1}, V_{2}, V_{3}\right]$ is a refinement of separation $\left[U_{0}, U_{1}, U_{2}\right]$. (b) Subgraphs $G\left[U_{0}\right], G\left(U_{1}\right)$, and $G\left(U_{2}\right)$ of $G$.

Property S1: $V_{0}, \ldots, V_{p}$ form a disjoint partition of $\operatorname{Node}(G)$.

Property S2: Any two $V_{i}$ and $V_{i^{\prime}}$ with $1 \leq i \neq i^{\prime} \leq p$ are not adjacent in $G$.

For instance, Figure 3(a) shows a separation $\left[V_{0}, V_{1}, V_{2}, V_{3}\right]$ of graph $G$ and Figure 4(a) shows another separation $\left[U_{0}, U_{1}, U_{2}\right]$ of $G$. For any subset $V$ of $\operatorname{Node}(G)$, let $G(V)$ be the subgraph of $G$ induced by $V \cup N b r_{G}(V)$ excluding the edges of $G\left[N b r_{G}(V)\right]$. If $\left[V_{0}, \ldots, V_{p}\right]$ is a separation of $G$, then $G\left[V_{0}\right], G\left(V_{1}\right), \ldots, G\left(V_{p}\right)$ form a disjoint partition of the edges of $G$. See Figures $3(\mathrm{~b})$ and 4 (b) for illustrations. Let $\log ^{(0)} n=n$. For any positive integer $k$, let $\log ^{(k)} n=\log \left(\log ^{(k-1)} n\right)$. For notational brevity, for any nonnegative integer $k$, let

$$
\ell_{k}=\max \left\{1, \log ^{(k)} n\right\} .
$$

For a nonnegative integer $k$, separation $\left[V_{0}, \ldots, V_{p}\right]$ of an $n$-node graph $G$ is a $k$-separation of $G$ if the following three properties hold.

Property S3: $\left|V_{0}\right|=o\left(\frac{n}{\ell_{k}}\right)$ and $p=o\left(\frac{n}{\ell_{k}}\right)+1$.

Property S4: $\left|V_{i}\right|+n b r_{G}\left(V_{i}\right)=\operatorname{poly}\left(\ell_{k}\right)$ holds for each $i=1, \ldots, p$.

Property S5: $\sum_{i=1}^{p} n b r_{G}\left(V_{i}\right)=o\left(\frac{n}{\ell_{k}}\right)$.

One can verify that $[\varnothing, \operatorname{Node}(G)]$ is a 0 -separation of $G$ 同 Let $\left[V_{0}, \ldots, V_{p}\right]$ and $\left[U_{0}, \ldots, U_{q}\right]$ be two separations of graph $G$. We say that $\left[V_{0}, \ldots, V_{p}\right]$ is a refinement of $\left[U_{0}, \ldots, U_{q}\right]$ if the following three properties hold.

Property R1: $U_{0} \subseteq V_{0}$.

Property R2: For each index $i=1, \ldots, p$, there is an index $j$ with $1 \leq j \leq q$ and $V_{i} \subseteq U_{j}$.

Property R3: For any indices $i, i^{\prime}, i^{\prime \prime}$ with $1 \leq i<i^{\prime}<i^{\prime \prime} \leq p$, if $V_{i} \cup V_{i^{\prime \prime}} \subseteq U_{j}$, then $V_{i^{\prime}} \subseteq U_{j}$.

\footnotetext{
${ }^{5}$ The " +1 " in Property S3 is redundant for $k \geq 1$. However, we need it so that $[\varnothing, \operatorname{Node}(G)]$ is a 0 separation of $G$, since $1 \neq o\left(\frac{n}{\ell_{0}}\right)$.
} 
For instance, in Figure $4(\mathrm{a}),\left[V_{0}, V_{1}, V_{2}, V_{3}\right]$ is a refinement of $\left[U_{0}, U_{1}, U_{2}\right]$. Below is the main lemma of the section.

Lemma 3.1. Let $k$ be a positive integer. Let $G$ be an n-node connected graph embedded on a genus-o $\left(n / \ell_{k}^{2}\right)$ surface. Given a $(k-1)$-separation $\mathbb{S}_{k-1}$ of $G$, it takes $O(n)$ time to compute a $k$-separation $\mathbb{S}_{k}$ of $G$ that is a refinement of $\mathbb{S}_{k-1}$.

The proof of Lemma 3.1 needs the following lemma, which can be proved by Lemmas 2.4 and 2.6.

Lemma 3.2. Let $k$ be a positive integer. Given an n-node graph $G$ embedded on a genus-o $\left(n / \ell_{k}^{2}\right)$ surface, it takes $O(n)$ time to compute an $o\left(\frac{n}{\ell_{k}}\right)$-node subset $V$ of $\operatorname{Node}(G)$ such that each node of $\operatorname{Node}(G) \backslash V$ has degree at most $\ell_{k}^{2}$ in $G$ and each connected component of $G \backslash V$ has at most $\ell_{k}^{4}$ nodes.

Proof. We first apply Lemma 2.6 to compute in $O(n)$ time an $o\left(\frac{n}{\ell_{k}}\right)$-node subset $V^{\prime}$ of Node $(G)$ such that $G \backslash V^{\prime}$ is planar. We then apply Lemma 2.4 to compute in $O(n)$ time a separator decomposition $\mathbb{T}$ of $G \backslash V^{\prime}$. For each vertex $S$ of $\mathbb{T}$, let $\operatorname{Offspring}(S)$ denote the union of all the vertices in the subtree of $\mathbb{T}$ rooted at $S$ and let offspring $(S)=\mid$ Offspring $(S) \mid$. Let $r=\ell_{k}^{2}$. Let $V^{\prime \prime}$ consist of the nodes of $G$ with degree more than $r$ in $G$. Let $V^{\prime \prime \prime}$ be the union of all the vertices $S$ of $\mathbb{T}$ with offspring $(S)>r^{2}$. Let $V=V^{\prime} \cup V^{\prime \prime} \cup V^{\prime \prime \prime}$. By $V^{\prime} \cup V^{\prime \prime \prime} \subseteq V$ and the definition of $\mathbb{T}$, each connected component of $G \backslash V$ has at most $r^{2}$ nodes. By $V^{\prime \prime} \subseteq V$, each node of Node $(G) \backslash V$ has degree at most $r$ in $G$. Since $G$ has $O(n)$ edges, $\left|V^{\prime \prime}\right|=O\left(\frac{n}{r}\right)=o\left(\frac{n}{\ell_{k}}\right)$. It remains to show $\left|V^{\prime \prime \prime}\right|=o\left(\frac{n}{\ell_{k}}\right)$. For each index $i \geq 1$, let $\mathbb{I}_{i}$ consist of the vertices $S$ of $\mathbb{T}$ with $r^{2} \cdot\left(\frac{3}{2}\right)^{i-1}<\operatorname{offspring}(S) \leq r^{2} \cdot\left(\frac{3}{2}\right)^{i}$. By $r^{2} \geq 1$ and $i \geq 1$, each $S \in \mathbb{I}_{i}$ is an internal vertex of $\mathbb{T}$. By definition of $\mathbb{T}$, we know that $O$ ffspring $(S)$ and Offspring $\left(S^{\prime}\right)$ are disjoint for any two distinct elements $S$ and $S^{\prime}$ of $\mathbb{I}_{i}$, implying that $\sum_{S \in \mathbb{I}_{i}}$ offspring $(S) \leq n$ holds. Since offspring $(S)>r^{2} \cdot(1.5)^{i-1}$ holds for each $S \in \mathbb{I}_{i}$, we have $\left|\mathbb{I}_{i}\right|<\frac{n}{r^{2} \cdot(1.5)^{i-1}}$. Since each $S \in \mathbb{I}_{i}$ is an internal vertex of $\mathbb{T}, S$ is a separator of $G[\operatorname{Offspring}(S)]$. Therefore, $|S|=O\left(r \cdot(1.5)^{i / 2}\right)$ holds for each vertex $S$ in $\mathbb{I}_{i}$. We have $\left|V^{\prime \prime \prime}\right|=\sum_{i \geq 1} \sum_{S \in \mathbb{I}_{i}}|S|=\sum_{i \geq 1} O\left(\frac{n}{r \cdot(1.5)^{i / 2}}\right)=O\left(\frac{n}{r}\right)=o\left(\frac{n}{\ell_{k}}\right)$. The lemma is proved.

Proof of Lemma 3.1 Suppose that $\left[U_{0}, \ldots, U_{q}\right]$ is the given $(k-1)$-separation $\mathbb{S}_{k-1}$. Let $V_{0}^{\prime}$ be the $O(n)$-time computable subset of $\operatorname{Node}(G)$ ensured by Lemma 3.2. We have $\left|V_{0}^{\prime}\right|=o\left(\frac{n}{\ell_{k}}\right)$. Let $V_{0}=U_{0} \cup V_{0}^{\prime}$. Let $\mathbb{C}$ consist of the connected components of $G \backslash V_{0}$. By $V_{0}^{\prime} \subseteq V_{0}$, each element of $\mathbb{C}$ has at most $\ell_{k}^{4}$ nodes. By $U_{0} \subseteq V_{0}$ and Properties S1 and S2 of $\mathbb{S}_{k-1}$, each element of $\mathbb{C}$ is contained by some $U_{j}$ with $1 \leq j \leq q$. For each $j=1, \ldots, q$, let $\mathbb{C}_{j}$ consist of the elements $C$ of $\mathbb{C}$ with $C \subseteq U_{j}$. We run Algorithm 1 to obtain (a) a disjoint partition $V_{1}, \ldots, V_{p}$ of $G \backslash V_{0}$ and (b) $p$ nodes hook $k_{1}, \ldots$, hook $k_{p}$ of $V_{0}$, which may not be distinct. Let $\mathbb{S}_{k}=\left[V_{0}, \ldots, V_{p}\right]$. Since $G$ is connected, each element of $\mathbb{C}$ is adjacent to $V_{0}$. The first statement of the outer repeat-loop is well defined. Since each element of $\mathbb{C}$ has at most $\ell_{k}^{4}$ nodes, the first statement of the inner repeat-loop is well defined. See Figure 5 for an illustration: Suppose that all nodes are in $U_{1}$. All nodes are initially 

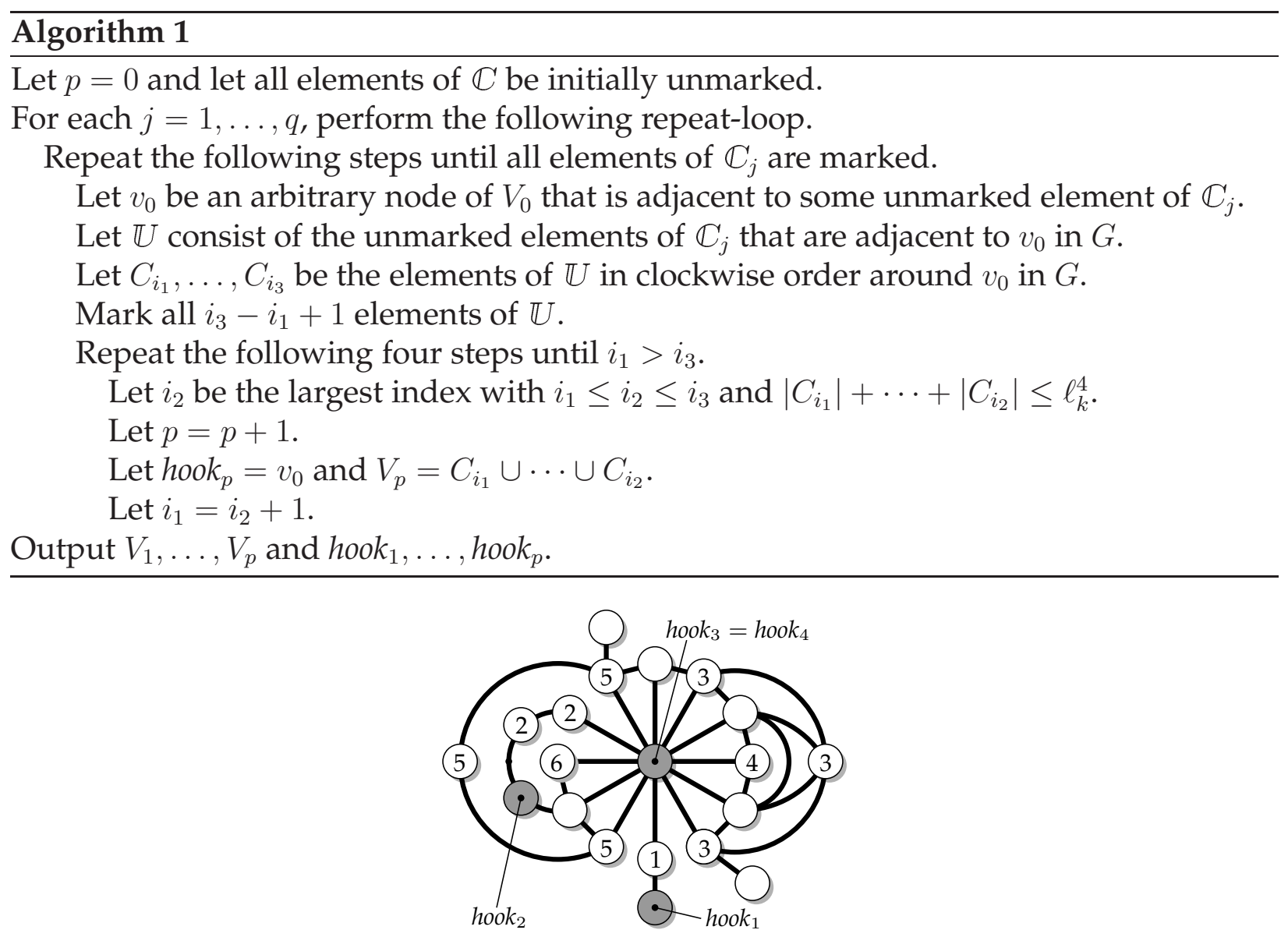

Figure 5: An illustration for Algorithm 1,

unmarked. Let $V_{0}$ consist of the nine unlabeled nodes, including the three gray nodes. For each $i=1, \ldots, 6$, let $C_{i}$ consist of the nodes with label $i$. That is, $C_{1}, \ldots, C_{6}$ are the six connected components of $G \backslash V_{0}$. Suppose that $\ell_{k}^{4}=7$ and the first two iterations of the outer repeat-loop obtain $V_{1}=C_{1}$ and $V_{2}=C_{2}$. In the third iteration of the outer repeatloop, $C_{3}, \ldots, C_{6}$ are the unmarked elements of $\mathbb{C}$ that are adjacent to hook $k_{3}$ in clockwise order around hook $k_{3}$. By $\left|C_{3}\right|+\left|C_{4}\right|+\left|C_{5}\right|=7$, the two iterations of the inner repeat-loop obtain $V_{3}=C_{3} \cup C_{4} \cup C_{5}$ and $V_{4}=C_{6}$.

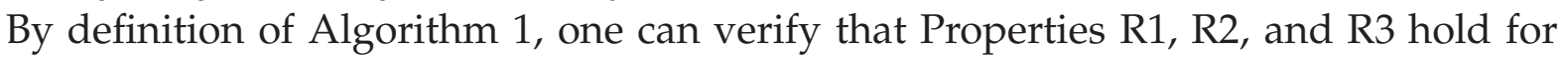
$\mathbb{S}_{k-1}$ and $\mathbb{S}_{k}$ (that is, $\mathbb{S}_{k}$ is a refinement of $\mathbb{S}_{k-1}$ ) and Properties S1 and S2 hold for $\mathbb{S}_{k}$. By Property $\mathbf{S 3}$ of $\mathbb{S}_{k-1}$, we have $\left|U_{0}\right|=o\left(\frac{n}{\ell_{k-1}}\right)=o\left(\frac{n}{\ell_{k}}\right)$. By $\left|V_{0}^{\prime}\right|=o\left(\frac{n}{\ell_{k}}\right)$, we have $\left|V_{0}\right| \leq$ $\left|U_{0}\right|+\left|V_{0}^{\prime}\right|=o\left(\frac{n}{\ell_{k}}\right)$. Let $I_{\text {small }}$ consist of the indices $i$ with $1 \leq i \leq p$ and $\left|V_{i}\right| \leq \frac{1}{2} \cdot \ell_{k}^{4}$. Let $I_{\text {large }}$ consist of the indices $i$ with $1 \leq i \leq p$ and $\left|V_{i}\right|>\frac{1}{2} \cdot \ell_{k}^{4}$. We show $p=\left|I_{\text {small }}\right|+\left|I_{\text {large }}\right|=o\left(\frac{n}{\ell_{k}}\right)$ as follows. By Property $\$ 1$ of $\mathbb{S}_{k}$, we have $\left|I_{\text {large }}\right|=o\left(\frac{n}{\ell_{k}}\right)$. To show $\left|I_{\text {small }}\right|=o\left(\frac{n}{\ell_{k}}\right)$, we categorize the indices $i$ in $I_{\text {small }}$ with $1 \leq i<p$ into the the following types, where $j$ is the index with $V_{i} \subseteq U_{j}$ : 


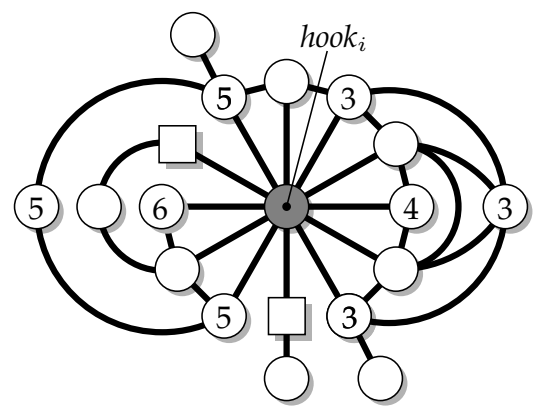

(a)

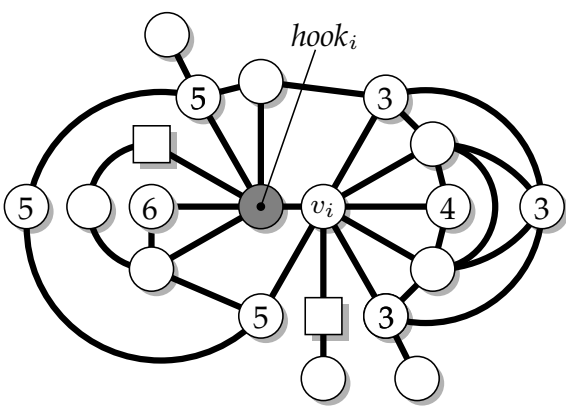

(b)

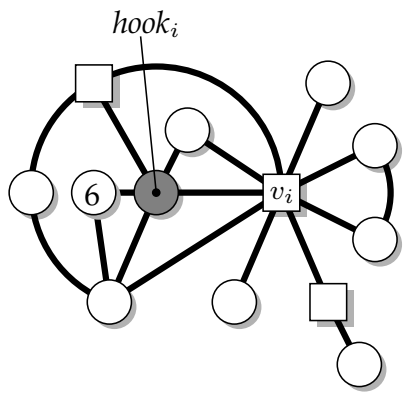

(c)

Figure 6: The operation that contracts all nodes of $V_{i}$ into a node $v_{i}$, which takes over some neighbors of hook .

Type 1: $i \in I_{\text {small }}$ and $i+1 \in I_{\text {large. }}$. The number of such indices $i$ is at most $\left|I_{\text {large }}\right|=o\left(\frac{n}{\ell_{k}}\right)$. Type 2: $i \in I_{\text {small }}$ and $i+1 \in I_{\text {small }}$.

Type 2a: $V_{i+1} \subseteq U_{j+1}$. The number of such indices $i$ is at most $q=o\left(\frac{n}{\ell_{k-1}}\right)=o\left(\frac{n}{\ell_{k}}\right)$.

Type 2b: $V_{i+1} \subseteq U_{j}$ and hook $k_{i} \in V_{0} \backslash U_{0}$. By Properties \$1 and S2 of $\mathbb{S}_{k-1}$, we know that $h_{o o k} \in U_{j}$. By definition of Algorithm $1, h_{0 o k^{\prime}} \neq$ hook $_{i}$ holds for all indices $i^{\prime}$ with $i<i^{\prime} \leq p$. The number of such indices $i$ is at most $\left|V_{0} \backslash U_{0}\right| \leq\left|V_{0}\right|=o\left(\frac{n}{\ell_{k}}\right)$.

Type 2c: $V_{i+1} \subseteq U_{j}$ and hook $\in U_{0}$. We have hook $k_{i} \in \mathrm{Nbr}_{G}\left(U_{j}\right)$. By definition of Algorithm 1, hook $i^{\prime} \neq$ hook $_{i}$ holds for all indices $i^{\prime}>i$ with $V_{i^{\prime}} \subseteq U_{j}$. By Property S5 of $\mathbb{S}_{k-1}$, the number of such indices $i$ is at most $\sum_{j=1}^{q} n b r_{G}\left(U_{j}\right)=o\left(\frac{n}{\ell_{k-1}}\right)=o\left(\frac{n}{\ell_{k}}\right)$.

We have $p=o\left(\frac{n}{\ell_{k}}\right)$. Property $\$ 3$ holds for $\mathbb{S}_{k}$. By definition of Algorithm 1, $\left|V_{i}\right| \leq \ell_{k}^{4}$ holds for each $i=1, \ldots, p$. By $V_{0}^{\prime} \subseteq V_{0}$, each node of $\operatorname{Node}(G) \backslash V_{0}$ has degree at most $\ell_{k}^{2}$. Property $\mathbb{S}_{4}$ holds for $\mathbb{S}_{k}$.

To see Property $\$ 5$ of $\mathbb{S}_{k}$, we obtain a contracted graph from $G$ by performing the following two steps for each $i=1, \ldots, p$ Step 1: Let $C_{i_{1}}, \ldots, C_{i_{2}}$ be the elements of $\mathbb{C}$ with $V_{i}=C_{i_{1}} \cup C_{i_{1}+1} \cup \cdots \cup C_{i_{2}}$ in clockwise order around hook $k_{i}$ in $G$. Split hook $k_{i}$ into two adjacent nodes hook $k_{i}$ and $v_{i}$ and let $v_{i}$ take over the neighbors of hook in clockwise order around hook $k_{i}$ from the first neighbor of hook $k_{i}$ in $C_{i_{1}}$ to the first neighbor of hook in $C_{i_{2}}$. Step 2: Contract all nodes of $V_{i}$ into node $v_{i}$ and delete multiple edges and self-loops. See Figure 6 for an illustration: For each $i=3, \ldots, 6$, let $C_{i}$ consist of the nodes with labels $i$ in Figure 6(a). Suppose that $i_{1}=3, i_{2}=5$, and $V_{i}=C_{3} \cup C_{4} \cup C_{5}$. The unlabeled circle nodes belong to $V_{0}$. The square nodes are two previously contracted nodes $v_{i^{\prime}}$ and $v_{i^{\prime \prime}}$ from $V_{i^{\prime}}$ and $V_{i^{\prime \prime}}$ for some indices $i^{\prime}$ and $i^{\prime \prime}$ with $1 \leq i^{\prime} \neq i^{\prime \prime}<i$. Figure 6(b) shows the result of Step 1. Figure 6 (c) shows the result of Step 2. Observe that each node that is adjacent to $V_{i}$ becomes a neighbor of $v_{i}$ after applying Steps 1 and 2. Also, each neighbor of hook $k_{i}$ that is not in $V_{i}$ either remains a neighbor of hook $k_{i}$ or becomes a neighbor of $v_{i}$ after applying

\footnotetext{
${ }^{6}$ The contraction procedure is only for proving Property 4 of $\mathbb{S}_{k}$, not needed for computing $\mathbb{S}_{k}$.
} 
Steps 1 and 2. Therefore, for each $i=1, \ldots, p$ and each node $v_{0} \in \operatorname{Nbr}_{G}\left(V_{i}\right)$, there is either an edge $\left(v_{0}, v_{i}\right)$ or an edge $\left(v_{i}, v_{i^{\prime}}\right)$ for some index $i^{\prime}$ with $i^{\prime}>i$ and hook $k_{i^{\prime}}=v_{0}$. Thus, $\sum_{i=1}^{p} n b r_{G}\left(V_{i}\right)$ is no more than the number of edges in the resulting contracted simple graph, which has $\left|V_{0}\right|+p=o\left(\frac{n}{\ell_{k}}\right)$ nodes. Observe that Step 1 does not increase the genus of the embedding. Since the subgraph induced by $V_{i} \cup\left\{v_{i}\right\}$ is connected, Step 2 does not increase the genus of the embedding, either. The number of edges in the resulting contracted simple genus-o $\left(n / \ell_{k}^{2}\right)$ graph is $o\left(\frac{n}{\ell_{k}}\right)$. Property 95 holds for $\mathbb{S}_{k}$. The lemma is proved.

\section{Our compression scheme}

This section proves Theorem 1.1 .

\subsection{Recovery string}

A labeling of graph $G$ is a one-to-one mapping from $\operatorname{Node}(G)$ to $\{0,1, \ldots, \operatorname{node}(G)-1\}$. For instance, Figure Z(a) shows a labeling for graph $G$. Let $G$ be a graph embedded on a surface. We say that a graph $\Delta$ embedded on the same surface is a triangulation of $G$ if $G$ is a subgraph of $\Delta$ with $\operatorname{Node}(\Delta)=\operatorname{Node}(G)$ such that each face of $\Delta$ has three nodes. The following lemma shows an $o(n)$-bit string with which the larger embedded labeled subgraphs of $G$ can be recovered from smaller embedded labeled subgraphs of $G$ in $O(n)$ time.

Lemma 4.1. Let $k$ be a positive integer. Let $G$ be an n-node graph embedded on a genus-o $\left(\frac{n}{\ell_{k}}\right)$ surface. Let $\Delta$ be a triangulation of $G$. Let $\mathbb{S}_{k}=\left[V_{0}, \ldots, V_{p}\right]$ be a given $k$-separation of $\Delta$ and $\mathbb{S}_{k-1}=\left[U_{0}, \ldots, U_{q}\right]$ be a given $(k-1)$-separation of $\Delta$ such that $\mathbb{S}_{k}$ is a refinement of $\mathbb{S}_{k-1}$. For any given labeling $L_{k, i}$ of $G\left(V_{i}\right)$ for each $i=1, \ldots, p$, the following statements hold.

1. It takes overall $O(n)$ time to compute a labeling $L_{k-1, j}$ of subgraph $G\left(U_{j}\right)$ for each $j=$ $1, \ldots, q$.

2. Given the above labelings $L_{k-1, j}$ of subgraphs $G\left(U_{j}\right)$ with $1 \leq j \leq q$, it takes $O(n)$ time to compute an o(n)-bit string $\operatorname{Rec}_{k}$ such that $G\left(U_{j}\right)$ and $L_{k-1, j}$ for all $j=1, \ldots, q$ can be recovered in overall $O(n)$ time from $\operatorname{Rec}_{k}$ and $G\left(V_{i}\right)$ and $L_{k, i}$ for all $i=1, \ldots, p$.

Proof. Since $\Delta$ is a subgraph $G$ with $\operatorname{Node}(\Delta)=\operatorname{Node}(G)$, one can easily verify that $\mathbb{S}_{k-1}$ (respectively, $\left.\mathbb{S}_{k}\right)$ is also a $(k-1)$-separation (respectively, $k$-separation) of $G$. For each $j=1, \ldots, q$, let $I_{j}$ consist of the indices $i$ with $V_{i} \subseteq U_{j}$. Let $W_{j}$ consist of the nodes of $G\left(U_{j}\right)$ that are not in any $V_{i}$ with $i \in I_{j}$. By Properties $\$ 1$ and $S 2$ of $\mathbb{S}_{k}, W_{j} \subseteq V_{0}$. For instance, if $G$ is as shown in Figure Z(a), where $v_{t}$ with $0 \leq t \leq 8$ denotes the node with label $t$. We have $I_{1}=\{1\}, I_{2}=\{2,3\}, W_{1}=\left\{v_{2}, v_{3}\right\}$, and $W_{2}=\left\{v_{0}, v_{1}, v_{2}, v_{6}\right\}$. Let the labeling $L_{k-1, j}$ for $G\left(U_{j}\right)$ be defined as follows. 


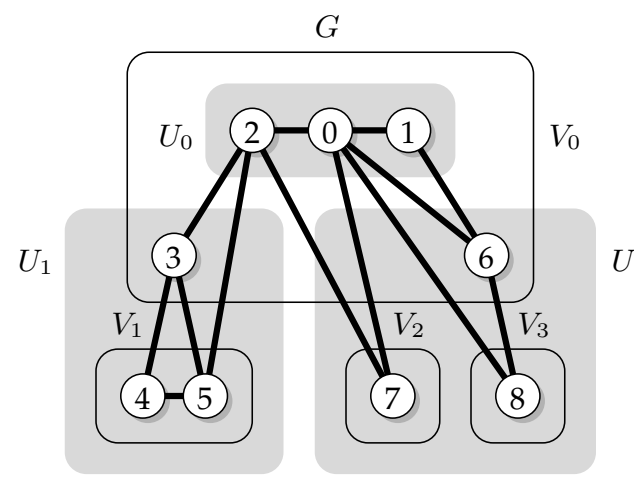

(a)

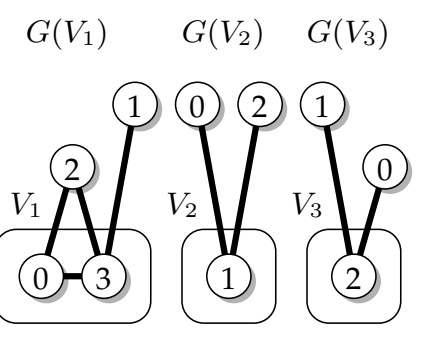

(b)

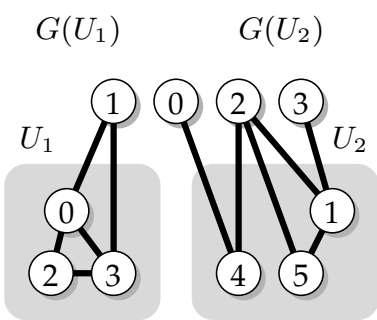

(c)

Figure 7: (a) Graph $G$ with a labeling. (b) Subgraphs $G\left(V_{1}\right), G\left(V_{2}\right)$, and $G\left(V_{3}\right)$ of $G$ with labelings. (c) Subgraphs $G\left(U_{1}\right)$ and $G\left(U_{2}\right)$ of $G$ with labelings.

- For the nodes of $G\left(U_{j}\right)$ in $W_{j}$, let $L_{k-1, j}$ be an arbitrary one-to-one mapping from $W_{j}$ to $\left\{0,1, \ldots,\left|W_{j}\right|-1\right\}$. In Figure Z( (c), we have $L_{k-1,1}\left(v_{2}\right)=1, L_{k-1,1}\left(v_{3}\right)=0$, $L_{k-1,2}\left(v_{0}\right)=2, L_{k-1,2}\left(v_{1}\right)=3, L_{k-1,2}\left(v_{2}\right)=0$, and $L_{k-1,2}\left(v_{6}\right)=1$.

- For the nodes of $G\left(U_{j}\right)$ not in $W_{j}$, let $L_{k-1, j}$ be the one-to-one mapping from $\bigcup_{i \in I_{j}} V_{i}$ to $\left\{\left|W_{j}\right|,\left|W_{j}\right|+1, \ldots\right.$, node $\left.\left(G\left(U_{j}\right)\right)-1\right\}$ obtained by sorting $\left(i, L_{k, i}(v)\right)$ for all indices $i \in I_{j}$ and all nodes $v \in V_{i}$ such that $L_{k-1, j}(v)<L_{k-1, j}\left(v^{\prime}\right)$ holds for a node $v$ of $V_{i}$ and a node $v^{\prime}$ of $V_{i^{\prime}}$ if and only if (a) $i<i^{\prime}$ or (b) $i=i^{\prime}$ and $L_{k, i}(v)<L_{k, i^{\prime}}\left(v^{\prime}\right)$. For instance, if $L_{k, 1}, L_{k, 2}$, and $L_{k, 3}$ are as shown in Figure $7(\mathrm{~b})$, then $L_{k-1,1}$ and $L_{k-1,2}$ can be as shown in Figure $7(\mathrm{c})$ and $L_{k-2,1}$ can be as shown in Figure $7(a)$.

It takes $O\left(\operatorname{node}\left(G\left(U_{j}\right)\right)\right)=O\left(\left|U_{j}\right|+n b r_{G}\left(U_{j}\right)\right)$ time to compute $L_{k-1, j}$ from all $L_{k, i}$ with $i \in I_{j}$. By Property $\$ 5$ of $\mathbb{S}_{k-1}$, it takes overall $O(n)$ time to compute all $L_{k-1, j}$ with $1 \leq j \leq q$ from all $L_{k, i}$ with $1 \leq i \leq p$. Statement 1 is proved.

By Property $\mathbb{S} 4$ of $\mathbb{S}_{k-1}$, the label of each node of $G\left(U_{j}\right)$ assigned by $L_{k-1, j}$ can be represented by $O\left(\log\right.$ poly $\left.\left(\ell_{k-1}\right)\right)=O\left(\ell_{k}\right)$ bits. By Property $\mathbb{S}_{4}$ of $\mathbb{S}_{k}$, the label of each node of $G\left(V_{i}\right)$ assigned by $L_{k, i}$ can be represented by $O\left(\log\right.$ poly $\left.\left(\ell_{k}\right)\right)=O\left(\ell_{k+1}\right)$ bits. For each index $j=1, \ldots, q$,

- string $\operatorname{Rec}_{k, j}^{\prime}$ stores the adjacency list of the embedded subgraph of $G\left(V_{j}\right)$ induced by $W_{j}$ via the labeling $L_{k-1, j}$ of $W_{j}$,

- string $\operatorname{Rec}_{k, j}^{\prime \prime}$ stores the information required to recover $L_{k-1, j}$ from all $L_{k, i}$ with $i \in I_{j}$, and

- string $\operatorname{Rec} c_{k, j}^{\prime \prime \prime}$ stores the information required to recover the embedding of $G\left(U_{j}\right)$ from the embeddings of all $G\left(V_{i}\right)$ with $i \in I_{j}$ and the embedding of the subgraph of $G\left(U_{j}\right)$ induced by $W_{j}$.

By definition of $W_{j}$, we have $\left|W_{j}\right|=\left|V_{0} \cap U_{j}\right|+n b r_{G}\left(U_{j}\right)$. It follows from Property S3 of 
$\mathbb{S}_{k}$ and Property $\mathbf{S} 5$ of $\mathbb{S}_{k-1}$ that

$$
\sum_{j=1}^{q}\left|W_{j}\right| \leq\left|V_{0}\right|+\sum_{j=1}^{q} n b r_{G}\left(U_{j}\right)=o\left(\frac{n}{\ell_{k}}\right)+o\left(\frac{n}{\ell_{k-1}}\right)=o\left(\frac{n}{\ell_{k}}\right) .
$$

Let $W=\bigcup_{j=1}^{q} W_{j}$. Since $G\left[V_{0}\right], G\left(V_{1}\right), \ldots, G\left(V_{p}\right)$ form a disjoint partition of the edges of $G$, the overall number of edges in the subgraphs of $G\left(V_{j}\right)$ induced by $W_{j}$ for all $j=1, \ldots, q$ is no more than the number of edges in $G[W]$, which is $O\left(|W|+o\left(\frac{n}{\ell_{k}}\right)\right) \leq O\left(\sum_{j=1}^{q}\left|W_{j}\right|\right)+$ $o\left(\frac{n}{\ell_{k}}\right)=o\left(\frac{n}{\ell_{k}}\right)$. Therefore,

$$
\sum_{j=1}^{q}\left\|\operatorname{Rec}_{k, j}^{\prime}\right\|=o\left(\frac{n}{\ell_{k}}\right) \cdot O\left(\ell_{k}\right)=o(n)
$$

It suffices for $\operatorname{Rec}_{k, j}^{\prime \prime}$ to store the list of $\left(i, L_{k, i}(v), L_{k-1, j}(v)\right)$ for all $i \in I_{j}$ and all $v \in \operatorname{Nbr}_{G}\left(V_{i}\right)$. By Property R3 of $\mathbb{S}_{k-1}$ and $\mathbb{S}_{k}$ and Property 4 of $\mathbb{S}_{k-1}$, index $i$ can be represented by an $O\left(\ell_{k}\right)$-bit offset $t$ such that $i$ is the $t$-th smallest index in $I_{j}$. Thus, $\left\|\operatorname{Rec}_{k, j}^{\prime \prime}\right\|=\sum_{i \in I_{j}} n b r_{G}\left(V_{i}\right)$. $O\left(\ell_{k}\right)$. By Property S5 of $\mathbb{S}_{k}$, we have $\sum_{j=1}^{q} \sum_{i \in I_{j}} n b r_{G}\left(V_{i}\right)=\sum_{i=1}^{p} n b r_{G}\left(V_{i}\right)=o\left(\frac{n}{\ell_{k}}\right)$. Therefore,

$$
\sum_{j=1}^{q}\left\|\operatorname{Rec}_{k, j}^{\prime \prime}\right\|=o\left(\frac{n}{\ell_{k}}\right) \cdot O\left(\ell_{k}\right)=o(n) .
$$

It suffices for $\operatorname{Rec}_{k, j}^{\prime \prime \prime}$ to store the list of $\left(L_{k-1, j}(v), L_{k-1, j}\left(v^{\prime}\right), L_{k-1, j}\left(v^{\prime \prime}\right)\right)$ for all pairs of edges $\left(v, v^{\prime}\right)$ and $\left(v, v^{\prime \prime}\right)$ of $G\left(U_{j}\right)$ such that (a) $v^{\prime \prime}$ is the neighbor of $v$ that immediately succeeds $v^{\prime}$ in clockwise order around $v$ in $G\left(U_{j}\right)$ and (b) nodes $v^{\prime}$ and $v^{\prime \prime}$ are not in the same partition of Node $\left(G\left(U_{j}\right)\right)$ formed by the $\left|I_{j}\right|+1$ disjoint sets $W_{j}$ and $V_{i}$ with $i \in I_{j}$. By Property $\$ 2$ of $\mathbb{S}_{k}$, node $v$ belongs to $W_{j} \subseteq V_{0}$. Since $\Delta$ is a triangulation of $G$, the neighbors of $v$ in $\Delta$ form a cycle that surrounds $v$ in $\Delta$. Let $P$ be the path of the cycle from $v^{\prime}$ to $v^{\prime \prime}$ in clockwise order around $v$. At least one node, say, $u$ of $P$ belongs to $V_{0}$, since otherwise Property $\mathrm{S} 2$ of $\mathbb{S}_{k}$ would imply that all nodes of $P$ belong to the same $V_{i}$ for some $i \in I_{j}$, contradicting with the choices of $v^{\prime}$ and $v^{\prime \prime}$. Edge $(v, u)$ belongs to $\Delta\left[V_{0}\right]$. Observe that each edge of $\Delta\left[V_{0}\right]$ can be identified by at most four such edge pairs $\left(v, v^{\prime}\right)$ and $\left(v, v^{\prime \prime}\right)$. Since the edges of $G\left(U_{j}\right)$ and $G\left(U_{j^{\prime}}\right)$ with $1 \leq j \neq j^{\prime} \leq q$ are disjoint, the number of edge pairs stored in $\operatorname{Rec}_{k, j}^{\prime \prime \prime}$ is at most four times the number of edges in $\Delta\left[V_{0}\right]$. By Property $\left.\$ 3\right]$ of $\mathbb{S}_{k}$ and the fact that $\Delta$ has genus $o\left(\frac{n}{\ell_{k}}\right)$, the number of edge pairs stored in $\operatorname{Rec}^{\prime \prime \prime}$ is $o\left(\frac{n}{\ell_{k}}\right)$. Therefore,

$$
\sum_{j=1}^{q}\left\|\operatorname{Rec}_{k, j}^{\prime \prime \prime}\right\|=o\left(\frac{n}{\ell_{k}}\right) \cdot O\left(\ell_{k}\right)=o(n)
$$

Let

$$
\begin{aligned}
\operatorname{Rec}_{k}^{\prime} & =\operatorname{Rec}_{k, 1}^{\prime} \circ \cdots \circ \operatorname{Rec}_{k, q}^{\prime}, \\
\operatorname{Rec}_{k}^{\prime \prime} & =\operatorname{Rec}_{k, 1}^{\prime \prime} \circ \cdots \circ \operatorname{Rec}_{k, q}^{\prime \prime}, \\
\operatorname{Rec}_{k}^{\prime \prime \prime} & =\operatorname{Rec}_{k, 1}^{\prime \prime \prime} \circ \cdots \circ \operatorname{Rec}_{k, q}^{\prime \prime \prime}, \\
\operatorname{Rec}_{k} & =\operatorname{Rec}_{k}^{\prime} \circ \operatorname{Rec}_{k}^{\prime \prime} \circ \operatorname{Rec}_{k}^{\prime \prime \prime} .
\end{aligned}
$$


By Equations (11), (2), and (3) and Lemma 2.2, we have $\left\|\operatorname{Rec}_{k}\right\|=o(n)$. It takes $O(n)$ time to compute $\operatorname{Rec}_{k}$ from all labelings $L_{k, j}$ and all embedded graphs $G\left(U_{j}\right)$ with $1 \leq j \leq q$ and all labelings $L_{k-1, i}$ and all embedded graphs $G\left(V_{i}\right)$ with $1 \leq i \leq p$. It also takes $O(n)$ time to recover all labelings $L_{k, j}$ and all embedded graphs $G\left(U_{j}\right)$ with $1 \leq j \leq q$ from $\operatorname{Rec}_{k}$ and all labelings $L_{k-1, i}$ and all embedded graphs $G\left(V_{i}\right)$ with $1 \leq i \leq p$. Statement 2 holds. The lemma is proved.

\subsection{Proving Theorem 1.1}

We are ready to prove the main theorem of the paper.

Proof of Theorem 1.1. Let $G \in \mathbb{G}$ be the $n$-node input graph embedded on a genus- $o\left(\frac{n}{\log ^{2} n}\right)$ surface. The encoding algorithm Encode $A$ performs the following four steps on $G$.

E1: Triangulate the embedded graph $G$ into a triangulation $\Delta$ of $G$. Let $\mathbb{S}_{0}$ be the 0 separation $[\varnothing, \operatorname{Node}(\Delta)]$ of $\Delta$. For each $k=1,2$, apply Lemma 3.1 to obtain a $k$ separation $\mathbb{S}_{k}$ of $\Delta$ that is a refinement of $\mathbb{S}_{k-1}$.

E2: Let $\left[V_{0}, \ldots, V_{p}\right]=\mathbb{S}_{2}$. Apply Lemma 2.3 with $\ell=\max _{1 \leq i \leq p}$ node $\left(G\left(V_{i}\right)\right)$ to compute (a) Label $(H)$ and $\operatorname{Optcode}(H)$ for all distinct graphs $H$ in class $\mathbb{G}$ with node $(H) \leq \ell$ and

(b) Table $(\mathbb{G}, \ell)$. For each $i=1, \ldots, p$, apply Lemma 2.3(1) to compute from Table $(\mathbb{G}, \ell)$ the binary string $\operatorname{Code}\left(V_{i}\right)=\operatorname{Optcode}\left(G\left(V_{i}\right)\right)$ and the labeling $L_{2, i}=\operatorname{Label}\left(G\left(V_{i}\right)\right)$.

E3: For each $k=2,1$, perform the following two substeps.

E[3 1: Let $\left[U_{0}, \ldots, U_{q}\right]=\mathbb{S}_{k-1}$ and $\left[V_{0}, \ldots, V_{p}\right]=\mathbb{S}_{k}$. For each $j=1, \ldots, q$, let binary string $\operatorname{Code}\left(U_{j}\right)=\operatorname{Code}\left(V_{i_{1}}\right) \circ \cdots \circ \operatorname{Code}\left(V_{i_{2}}\right)$, where $\left\{i_{1}, i_{1}+1, \ldots, i_{2}\right\}$ are the indices $i$ with $V_{i} \subseteq U_{j}$.

E33 2: Apply Lemma 4.11(1) to obtain the labelings $L_{k-1, j}$ of subgraphs $G\left(U_{j}\right)$ for all $j=1, \ldots, q$. Apply Lemma 4.1(2) to obtain the $o(n)$-bit binary string $\operatorname{Rec}_{k}$.

E4: By $\mathbb{S}_{0}=[\varnothing, \operatorname{Node}(G)]$, now we have Code $\left(\operatorname{Node}(G)\right.$ ) (and a labeling $L_{0,1}$ for $G=$ $G(\operatorname{Node}(G)))$. The output binary string $\operatorname{Code}_{A}(G)=\operatorname{Code}(\operatorname{Node}(G)) \circ \operatorname{Table}(\mathbb{G}, \ell) \circ \operatorname{Rec}_{1} \circ$ $\mathrm{ReC}_{2}$.

By Lemma 3.1, Step E1 takes $O(n)$ time. By Property S5 of $\mathbb{S}_{2}$, we have $\sum_{i=1}^{p}$ node $\left(G\left(V_{i}\right)\right)=$ $n+o(n)$. By Lemma 2.3, Step E2 takes $O(n)$ time. By Lemmas 2.2 and 4.1, Step E3 takes $O(n)$ time. By Lemma 2.2, Step $\mathrm{E} 4$ takes $O(n)$ time. Therefore, the encoding algorithm Encode $_{A}(G)$ runs in $O(n)$ time. Condition C1 holds.

The decoding algorithm Decode $_{A}$ performs the following five steps on $\operatorname{Code}_{A}(G)$.

D1: Obtain Code $(\operatorname{Node}(G))$, Table $(\mathbb{G}, \ell), \operatorname{Rec}_{1}$, and $\operatorname{Rec}_{2}$ from $\operatorname{Code}_{A}(G)$.

$D 2$ : Let $\mathbb{S}_{0}=[\varnothing, \operatorname{Node}(G)]$. For each $k=1,2$, perform the following substep.

D2.1: Let $\left[U_{0}, \ldots, U_{q}\right]=\mathbb{S}_{k-1}$ and $\left[V_{0}, \ldots, V_{p}\right]=\mathbb{S}_{k}$. For each $j=1, \ldots, q$, obtain all $\operatorname{Code}\left(V_{i}\right)$ with $V_{i} \subseteq U_{j}$ from Code $\left(U_{j}\right)$. 
D3: Let $\left[V_{0}, \ldots, V_{p}\right]=\mathbb{S}_{2}$. Apply Lemma 2.3(2) to obtain $G\left(V_{i}\right)$ and $L_{2, i}=\operatorname{Label}\left(G\left(V_{i}\right)\right)$ from $\operatorname{Code}\left(V_{i}\right)=\operatorname{Optcode}\left(G\left(V_{i}\right)\right)$ and Table $(\mathbb{G}, \ell)$ for each $i=1, \ldots, p$.

$D 4$ : For each $k=2,1$, perform the following substep.

D4.1: Let $\left[U_{0}, \ldots, U_{q}\right]=\mathbb{S}_{k-1}$ and $\left[V_{0}, \ldots, V_{p}\right]=\mathbb{S}_{k}$. Apply Lemma 4.1(2) to recover $G\left(U_{j}\right)$ and $L_{k-1, j}$ with $1 \leq j \leq q$ from $G\left(V_{i}\right)$ and $L_{k, i}$ with $1 \leq i \leq p$ and $\operatorname{Rec}_{k}$.

D5: Output $G=G(\operatorname{Node}(G))$.

By Lemma 2.2, Step D1 takes $O(n)$ time. By Lemma 2.2, Step D2 takes $O(n)$ time. By Property S5 of $\mathbb{S}_{2}$, we have $\sum_{i=1}^{p} \operatorname{node}\left(G\left(V_{i}\right)\right)=n+o(n)$. By Lemma 2.3(2), Step D3 takes $O(n)$ time. By Lemma 4.1(2), Step D4 takes $O(n)$ time. Therefore, the decoding

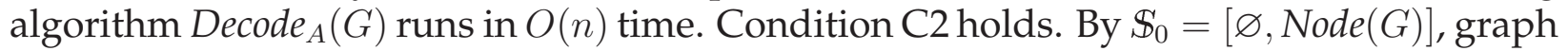
$G=G(\operatorname{Node}(G))$ is correctly recovered from $\operatorname{Code}_{A}(G)$ at the end of Step D4. Therefore, $A$ is a compression scheme for $\mathbb{G}$.

To show Condition C3, we first prove the following claim for each $k=1,2$.

Claim 1. Suppose that $\left[U_{0}, \ldots, U_{q}\right]=\mathbb{S}_{k-1}$ and $\left[V_{0}, \ldots, V_{p}\right]=\mathbb{S}_{k}$. If $\sum_{i=1}^{p}\left\|\operatorname{Code}\left(V_{i}\right)\right\| \leq \beta n+$ $o(n)$ and $\left\|\operatorname{Code}\left(V_{i}\right)\right\|=$ poly $\left(\ell_{k}\right)$ holds for each $i=1, \ldots, p$, then $\sum_{j=1}^{q}\left\|\operatorname{Code}\left(U_{j}\right)\right\| \leq \beta n+o(n)$ and $\left\|\operatorname{Code}\left(U_{j}\right)\right\|=\operatorname{poly}\left(\ell_{k-1}\right)$ holds for each $j=1, \ldots, q$.

Proof of Claim 1 For each $j=1,2, \ldots, q$, let $I_{j}$ consist of the indices $i$ with $V_{i} \subseteq U_{j}$. By Property $\mathbb{S} 4$ of $\mathbb{S}_{k-1}$, we have $\left|I_{j}\right| \leq\left|U_{j}\right|=\operatorname{poly}\left(\ell_{k-1}\right)$. Therefore, $\sum_{i \in I_{j}}\left\|\operatorname{Code}\left(V_{i}\right)\right\|=$ poly $\left(\ell_{k-1}\right)$, implying $O\left(\log \sum_{i \in I_{j}}\left\|\operatorname{Code}\left(V_{i}\right)\right\|\right)=O\left(\ell_{k}\right)$. By Property $\mathbb{S}_{3}$ of $\mathbb{S}_{k}, \sum_{j=1}^{q}\left|I_{j}\right|=$ $p=O\left(\frac{n}{\ell_{k}}\right)$. By Lemma2.2, we have

$$
\sum_{j=1}^{q}\left\|\operatorname{Code}\left(U_{j}\right)\right\|=\sum_{j=1}^{q} O\left(\left|I_{j}\right| \cdot \ell_{k}\right)+\sum_{i=1}^{p}\left\|\operatorname{Code}\left(V_{i}\right)\right\| \leq \beta n+o(n) .
$$

We also have

$$
\left\|\operatorname{Code}\left(U_{j}\right)\right\|=O\left(\left|I_{j}\right| \cdot \ell_{k}\right)+\sum_{i \in I_{j}}\left\|\operatorname{Code}\left(V_{i}\right)\right\|=\operatorname{poly}\left(\ell_{k-1}\right) .
$$

The claim is proved.

Let $\left[V_{0}, \ldots, V_{p}\right]=\mathbb{S}_{2}$. For each $i=1, \ldots, p$, let $n_{i}=\operatorname{node}\left(G\left(V_{i}\right)\right)=\left|V_{i}\right|+n b r_{G}\left(V_{i}\right)$. By Property $\mathbb{S} 5$ of $\mathbb{S}_{2}$, we have $\sum_{i=1}^{p} n_{i}=n+o(n)$. By Step E2 of Encode $A_{A}(G)$ and Lemma 2.3, we have $\left\|\operatorname{Code}\left(V_{i}\right)\right\|=\left\|\operatorname{Optcode}\left(G\left(V_{i}\right)\right)\right\|=\left\lceil\log n u m\left(\mathbb{G}, n_{i}\right)\right\rceil \leq \beta n_{i}+o\left(n_{i}\right)$. By Property $\mathbb{S} 4$ of $\mathbb{S}_{2}$ and the assumption that $\log n u m(\mathbb{G}, n)=O(n)$,

$$
\left\|\operatorname{Code}\left(V_{i}\right)\right\|=\operatorname{poly}\left(\ell_{2}\right)
$$

holds for each $i=1,2, \ldots, p$. We have

$$
\sum_{i=1}^{p}\left\|\operatorname{Code}\left(V_{i}\right)\right\| \leq \sum_{i=1}^{p}\left(\beta n_{i}+o\left(n_{i}\right)\right)=\beta \cdot(n+o(n))+o(\beta n+o(n))=\beta n+o(n) .
$$


Combining Equations (4) and (5), Claim 1 for $k=2,1$, and $\mathbb{S}_{0}=[\varnothing, \operatorname{Node}(G)]$, we have $\|\operatorname{Code}(\operatorname{Node}(G))\| \leq \beta n+o(n)$. By Lemma 2.2 and $\|\operatorname{Table}(\mathbb{G}, \ell)\|+\left\|\operatorname{Rec}_{1}\right\|+\left\|\operatorname{Rec}_{2}\right\|=o(n)$, we have $\left\|\operatorname{Code}_{A}(G)\right\| \leq \beta n+o(n)$. Condition C3 holds. The theorem is proved.

\section{Extension}

This section proves Theorem 1.2 The only place in our proof of Theorem 1.1 requiring $\mathbb{G}$ to be hereditary is Step E2, We need $G\left(V_{i}\right) \in \mathbb{G}$ so that $\operatorname{Optcode}\left(G\left(V_{i}\right)\right)$ and Label $\left(G\left(V_{i}\right)\right)$ can be obtained from Table $(\mathbb{G}, \ell)$. For a non-hereditary class $\mathbb{G}$, we can substitute $G\left(V_{i}\right)$ by a graph $H_{i} \in \mathbb{G}$ that is close to $G\left(V_{i}\right)$ for each $i=1,2, \ldots, p$ as long as the overall number of bits required to encode the overall difference between $G\left(V_{i}\right)$ and $H_{i}$ is $o(n)$. The following corollary is an example of such an extension.

Corollary 5.1. Let $\mathbb{G}$ be a class of graphs satisfying $\log n u m(\mathbb{G}, n)=O(n)$ and that any input $n$ node graph $G \in \mathbb{G}$ to be encoded comes with a genus-o $\left(\frac{n}{\log ^{2} n}\right)$ embedding. If for any 2-separation $\left[V_{0}, \ldots, V_{p}\right]$ of any graph $G \in \mathbb{G}$, there exist graphs $H_{1}, \ldots, H_{p}$ in $\mathbb{G}$ such that each $G\left(V_{i}\right)$ with $1 \leq i \leq p$ can be obtained from $H_{i}$ by first deleting $O\left(n b r_{G}\left(V_{i}\right)\right)$ nodes (together with their incident edges) and then updating (adding or deleting) $O\left(n b r_{G}\left(V_{i}\right)\right)$ edges, then $\mathbb{G}$ admits an optimal compression scheme.

Proof. We revise algorithm Encode $_{A}$ by updating Steps $\mathrm{E} 2$ and $\mathrm{E} 4$ as follows.

E2': Let $\left[V_{0}, \ldots, V_{p}\right]=\mathbb{S}_{2}$. Compute $H_{1}, \ldots, H_{p}$ from $G\left(V_{1}\right), \ldots, G\left(V_{p}\right)$. Apply Lemma 2.3 with $\ell=\max _{1 \leq i \leq p} \operatorname{node}\left(H_{i}\right)$ to compute (a) Label $(H)$ and Optcode $(H)$ for each distinct graph $H \in \mathbb{G}$ with $\operatorname{node}(H) \leq \ell$ and $(\mathrm{b}) \operatorname{Table}(\mathbb{G}, \ell)$. Apply Lemma 2.3 (1) to compute $\operatorname{Code}\left(V_{i}\right)=\operatorname{Optcode}\left(H_{i}\right)$ and $L_{2, i}^{\prime}=\operatorname{Label}\left(H_{i}\right)$ from Table $(\mathbb{G}, \ell)$ for all indices $i=1, \ldots, p$. Let $L_{2, i}$ be the labeling of $G\left(V_{i}\right)$ obtained from the labeling $L_{2, i}^{\prime}$ of $H_{i}$ such that if $v$ and $v^{\prime}$ are two distinct nodes of $G\left(V_{i}\right)$ with $L_{2, i}^{\prime}(v)<L_{2, i}^{\prime}\left(v^{\prime}\right)$, then we have $L_{2, i}(v)<L_{2, i}\left(v^{\prime}\right)$. Let Fix be the binary string storing the difference between $H_{i}$ and $G\left(V_{i}\right)$ via labeling $L_{2, i}^{\prime}$. Let Fix $=$ Fix $_{1} \circ \cdots \circ$ Fix $_{p}$.

$E 4^{\prime}$ : By $\mathbb{S}_{0}=[\varnothing, \operatorname{Node}(G)]$, now we have $\operatorname{Code}\left(\operatorname{Node}(G)\right.$ ) (and a labeling $L_{0,1}$ for $G=$ $G(\operatorname{Node}(G)))$. The output binary string $\operatorname{Code}_{A}(G)$ for $G$ is $\operatorname{Code}(\operatorname{Node}(G)) \circ \operatorname{Table}(\mathbb{G}, \ell) \circ$ $\operatorname{Rec}_{1} \circ \operatorname{Rec}_{2} \circ \mathrm{Fix}$.

By $O(1)^{\text {poly }(\ell)}=o(n)$, it takes $o(n)$ time to compute an $o(n)$-bit string Table ${ }^{\prime}$ such that graphs $H_{1}, \ldots, H_{p}$ satisfying the above conditions can be obtained from $G\left(V_{1}\right), \ldots, G\left(V_{p}\right)$ and Table in $O(n)$ time. By Property S5 of $\mathbb{S}_{2}$ and the conditions of $H_{1}, \ldots, H_{p}$, we have $\sum_{i=1}^{p} \operatorname{node}\left(G\left(V_{i}\right)\right) \leq \sum_{i=1}^{p}$ node $\left(H_{i}\right)=n+o(n)$. By Lemmas 2.2 and 2.3. Step E[2 takes $O(n)$ time. By Lemma2.2, Step $\mathrm{E} 4$ takes $O(n)$ time. Therefore, Condition C1 holds for the revised Encode ${ }_{A}$. We revise algorithm Decode ${ }_{A}$ by updating Steps D[1] and D[3 as follows.

$D 1$ ': Obtain Code $(G)$, Table $(\mathbb{G}, \ell), \operatorname{Rec}_{1}, \operatorname{Rec}_{2}$, and Fix from $\operatorname{Code}_{A}(G)$. 


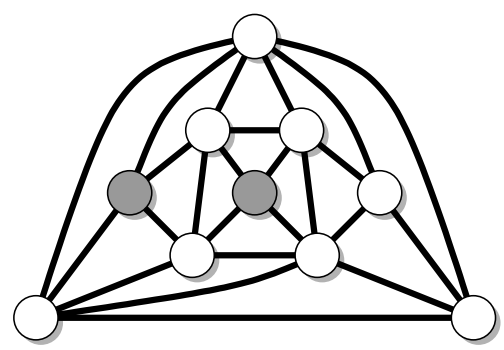

(a)

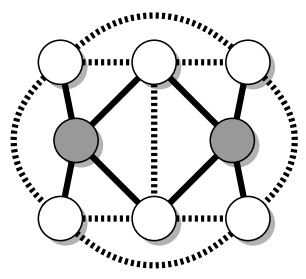

(b)

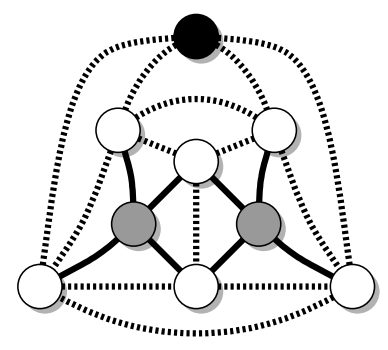

(c)

Figure 8: (a) A plane triangulation $\Delta$, where $V_{i}$ consists of the gray nodes. (b) The subgraph $\Delta\left[V_{i}\right]$, where the dotted edges are those in $\Delta\left[V_{i}\right] \backslash \Delta\left(V_{i}\right)$. (c) A plane triangulation $H_{i}$ obtained by adding the dark node $v_{F}$ and four edges in the external face $F$ of $H_{i}$.

D3': Let $\left[V_{0}, \ldots, V_{p}\right]=\mathbb{S}_{2}$. Apply Lemma 2.3(2) to obtain $H_{i}$ and $L_{2, i}^{\prime}=\operatorname{Label}\left(H_{i}\right)$ from $\operatorname{Code}\left(V_{i}\right)=\operatorname{Optcode}\left(H_{i}\right)$ and Table $(\mathbb{G}, \ell)$ for each $i=1, \ldots, p$. Apply Lemma 2.2 to obtain all Fix $i_{i}$ with $1 \leq i \leq p$ from Fix. Obtain $G\left(V_{i}\right)$ and $L_{2, i}$ from Fix $x_{i}, H_{i}$, and $L_{2, i}^{\prime}$ for all $i=1,2, \ldots, p$.

Both revised steps take $O(n)$ time. Condition C2 holds for the revised Decode ${ }_{A}$. Subgraph $G\left(V_{i}\right)$ can be obtained from $H_{i}$ by first deleting $O\left(n b r_{G}\left(V_{i}\right)\right)$ nodes (and their incident edges) and then updating $O\left(n b r_{G}\left(V_{i}\right)\right)$ edges. It follows from Property $\mathrm{S} 4$ of $\mathbb{S}_{2}$ that $\left\|F i x_{i}\right\|=O\left(n b r_{G}\left(V_{i}\right) \cdot \ell_{2}\right)$. By Property S5 of $\mathbb{S}_{2}$, we have $\sum_{i=1}^{p}\left\|F i x_{i}\right\|=o\left(\frac{n}{\ell_{2}}\right) \cdot O\left(\ell_{2}\right)=o(n)$. By Lemma 2.2, we have $\|F i x\|=o(n)$. Condition C3 holds the revised $\operatorname{Code}_{A}(G)$. The corollary is proved.

We use Corollary 5.1 to prove Theorem 1.2 .

Proof of Theorem 1.2 Let $\Delta$ be an $n$-node triangulation of a genus- $g$ surface. Let $\left[V_{0}, \ldots, V_{p}\right]$ be a 2-separation of $\Delta$. Let $\mathbb{F}$ consist of the non-triangle faces of $\Delta\left[V_{i}\right]$. Let $H_{i}$ be the plane triangulation obtained from $\Delta\left[V_{i}\right]$ by performing the following two steps for each face $F \in \mathbb{F}$ : (1) Add a node $v_{F}$ in $F$. (2) For each node $u$ on the boundary of $F$, add an edge $\left(u, v_{F}\right)$. See Figure 8 for an illustration. Since $\Delta$ is a triangulation, the boundary of $F$ contains at least two nodes $u$ with $\operatorname{Nbr}_{\Delta}(u) \nsubseteq \operatorname{Node}\left(\Delta\left(V_{i}\right)\right)$. Therefore, at least two nodes of $\operatorname{Nbr}\left(V_{i}\right)$ belong to the boundary of $F$. Let $e_{F}$ be an edge between two arbitrary nodes of $\operatorname{Nbr}\left(V_{i}\right)$ that belong to the boundary of $F$. The union of $e_{F}$ over all faces $F \in \mathbb{F}$ has genus no more than $g=O(1)$. Therefore, the number of added nodes to triangulate $\Delta\left[V_{i}\right]$ is $O\left(n b r_{\Delta}\left(V_{i}\right)\right)$. The number of edges in $\Delta\left[V_{i}\right] \backslash \Delta\left(V_{i}\right)$ is also $O\left(n b r_{\Delta}\left(V_{i}\right)\right)$. Thus, $\Delta\left(V_{i}\right)$ can be obtained from $H_{i}$ by first deleting $O\left(n b r_{\Delta}\left(V_{i}\right)\right)$ nodes together with their incident edges and then deleting $O\left(n b r_{\Delta}\left(V_{i}\right)\right)$ edges. By Corollary 5.1, Statement 1 is proved.

Let $G$ be an $n$-node floorplan. Since each node of $G$ has at most three neighbors in $G$, one can easily obtain a floorplan $H_{i}$ from $G\left(V_{i}\right)$ by adding $O\left(n b r_{G}\left(V_{i}\right)\right)$ nodes and edges. See Figure 9 for an example. Statement 2 follows from Corollary 5.1 . 


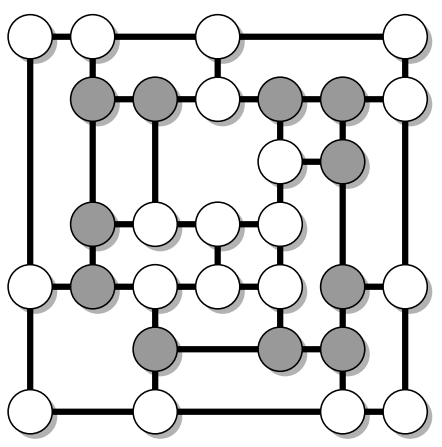

(a)

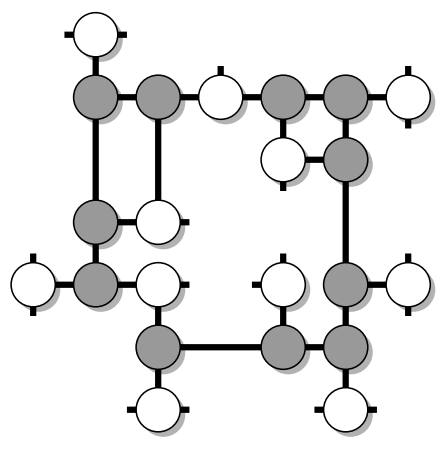

(b)

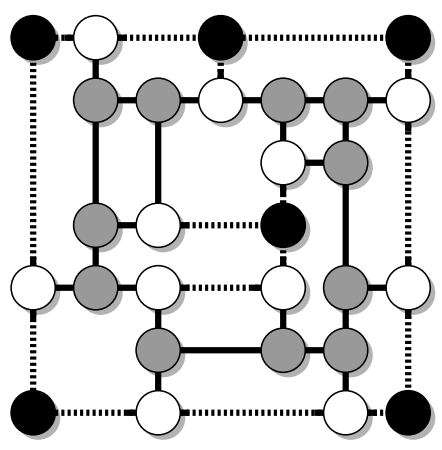

(c)

Figure 9: (a) A floorplan $G$, where $V_{i}$ consists of the gray nodes. (b) The subgraph $G\left(V_{i}\right)$. (c) A floorplan $H_{i}$ obtained from $G\left(V_{i}\right)$ by adding $O\left(n b r_{G}\left(V_{i}\right)\right)$ nodes and edges.

\section{Concluding remarks}

Our optimal compression schemes rely on a linear-time obtainable embedding. Can this requirement be dropped? It would be of interest to extend our compression schemes to support efficient queries and updates. We leave open the problems of obtaining optimal compression schemes for $O(1)$-connected genus- $O(1)$ graphs and 3D floorplans [23, 60, 86, 59, 98, 99, 61, 22].

\section{References}

[1] I. Abraham, D. Malkhi, and D. Ratajczak. Compact multicast routing. In Proceedings of the 23rd International Symposium on Distributed Computing, Lecture Notes in Computer Science 5805, pages 364-378, 2009.

[2] M. Adler and M. Mitzenmacher. Towards compressing web graphs. In Proceedings of the 11th Data Compression Conference, pages 203-212. IEEE, 2001.

[3] R. Agarwal, P. B. Godfrey, and S. Har-Peled. Approximate distance queries and compact routing in sparse graphs. In Proceedings of the 30th IEEE International Conference on Computer Communications, pages 1754-1762, 2011.

[4] A. R. Agnihotri, S. Ono, and P. H. Madden. An effective approach for large scale floorplanning. In Proceedings of the 20th ACM Great Lakes Symposium on VLSI, pages 107-110, 2010.

[5] V. N. Anh and A. Moffat. Local modeling for webgraph compression. In Proceedings of the Data Compression Conference, page 519. IEEE Computer Society, 2010.

[6] S. R. Arikati, A. Maheshwari, and C. D. Zaroliagis. Efficient computation of implicit representations of sparse graphs. Discrete Applied Mathematics, 78(1-3):1-16, 1997.

[7] Y. Asano, Y. Miyawaki, and T. Nishizeki. Efficient compression of web graphs. IEICE Transactions, 92-A(10):2454-2462, 2009. 
[8] P. Banerjee, S. Sur-Kolay, and A. Bishnu. Fast unified floorplan topology generation and sizing on heterogeneous FPGAs. IEEE Transactions on CAD of Integrated Circuits and Systems, 28(5):651-661, 2009.

[9] J. Barbay, L. C. Aleardi, M. He, and J. I. Munro. Succinct representation of labeled graphs. In T. Tokuyama, editor, Proceedings of the 18th International Symposium on Algorithms and Computation, pages 316-328, 2007.

[10] T. C. Bell, J. G. Cleary, and I. H. Witten. Text Compression. Prentice-Hall, Englewood Cliffs, NJ, 1990.

[11] D. Benoit, E. D. Demaine, J. I. Munro, R. Raman, V. Raman, and S. S. Rao. Representing trees of higher degree. Algorithmica, 43(4):275-292, 2005.

[12] S. N. Bhatt and F. T. Leighton. A framework for solving VLSI graph layout problems. Jouranl of Computer and System Sciences, 28(2):300-343, 1984.

[13] D. K. Blandford, G. E. Blelloch, and I. A. Kash. Compact representations of separable graphs. In Proceedings of the 14th Annual ACM-SIAM Symposium on Discrete Algorithms, pages 679688, 2003.

[14] G. E. Blelloch and A. Farzan. Succinct representations of separable graphs. In A. Amir and L. Parida, editors, Proceedings of the 21st Annual Symposium on Combinatorial Pattern Matching, Lecture Notes in Computer Science 6129, pages 138-150. Springer, 2010.

[15] A. Z. Broder, R. Kumar, F. Maghoul, P. Raghavan, S. Rajagopalan, R. Stata, A. Tomkins, and J. L. Wiener. Graph structure in the Web. Computer Networks, 33(1-6):309-320, 2000.

[16] S. Chechik. Fault-tolerant compact routing schemes for general graphs. In L. Aceto, M. Henzinger, and J. Sgall, editors, Proceedings of the 38th International Colloquium on Automata, Languages and Programming, Lecture Notes in Computer Science 6756, pages 101-112, 2011.

[17] T.-C. Chen and Y.-W. Chang. Modern floorplanning based on $B^{*}$-tree and fast simulated annealing. IEEE Transactions on CAD of Integrated Circuits and Systems, 25(4):637-650, 2006.

[18] Y.-T. Chiang, C.-C. Lin, and H.-I. Lu. Orderly spanning trees with applications. SIAM Journal on Computing, 34(4):924-945, 2005.

[19] P.-Y. Chuang. Improved compact encoding of rectangular drawings. Master's thesis, Department of Computer Science and Information Engineering, National Taiwan University, 2008.

[20] R. C.-N. Chuang, A. Garg, X. He, M.-Y. Kao, and H.-I. Lu. Compact encodings of planar graphs via canonical ordering and multiple parentheses. In K. G. Larsen, S. Skyum, and G. Winskel, editors, Proceedings of the 25th International Colloquium on Automata, Languages, and Programming, Lecture Notes in Computer Science 1443, pages 118-129, Aalborg, Denmark, 1998. Springer-Verlag.

[21] F. Claude and G. Navarro. Fast and compact web graph representations. ACM Transactions on the Web, 4(4), 2010. 
[22] J. Cong and Y. Ma. Thermal-aware 3D floorplan. In Y. Xie, J. Cong, and S. Sapatnekar, editors, Three Dimensional Integrated Circuit Design, pages 63-102. Springer, 2010.

[23] D. Cuesta, J. Ayala, J. Hidalgo, M. Poncino, A. Acquaviva, and E. Macii. Thermal-aware floorplanning exploration for 3D multi-core architectures. In R. I. Bahar, F. Lombardi, D. Atienza, and E. Brunvand, editors, Proceedings of the 20th ACM Great Lakes Symposium on VLSI 2009, pages 99-102. ACM, 2010.

[24] P. Dasgupta and S. Sur-Kolay. Slicible rectangular graphs and their optimal floorplans. ACM Transactions on Design Automation of Electronic Systems, 6(4):447-470, 2001.

[25] N. Deo and B. Litow. A structural approach to graph compression. In Proceedings of MFCS'98 Satellite Workshop on Communications, pages 91-101, 1998.

[26] H. N. Djidjev and S. M. Venkatesan. Planarization of graphs embedded on surfaces. In Proceedings of the 21st Workshop on Graph-Theoretic Concepts in Computer Science, Lecture Notes in Computer Science 1017, pages 62-72. Springer, 1995.

[27] P. Elias. Universal codeword sets and representations of the integers. IEEE Transactions on Information Theory, IT-21:194-203, 1975.

[28] A. Farzan and J. I. Munro. Succinct representations of arbitrary graphs. In D. Halperin and K. Mehlhorn, editors, Proceedings of the 16th Annual European Symposium, Lecture Notes in Computer Science 5193, pages 393-404. Springer, 2008.

[29] A. Farzan and J. I. Munro. A uniform approach towards succinct representation of trees. In Proceedings of the 11th Scandinavian Workshop on Algorithm Theory, Lecture Notes in Computer Science 5124, pages 173-184. Springer, 2008.

[30] A. Farzan, R. Raman, and S. S. Rao. Universal succinct representations of trees? In S. Albers, A. Marchetti-Spaccamela, Y. Matias, S. E. Nikoletseas, and W. Thomas, editors, Proceedings of the 36th International Colloquium on Automata, Languages and Programming, Lecture Notes in Computer Science 5555, pages 451-462. Springer, 2009.

[31] T. Feder and R. Motwani. Clique partitions, graph compression and speeding-up algorithms. Journal of Computer and System Sciences, 51(2):261-272, 1995.

[32] Y. Feng and D. P. Mehta. Module relocation to obtain feasible constrained floorplans. IEEE Transactions on CAD of Integrated Circuits and Systems, 25(5):856-866, 2006.

[33] R. Fujimaki and T. Takahashi. A surjective mapping from permutations to room-to-room floorplans. IEICE Transactions on Fundamentals of Electronics, Communications and Computer Sciences, 90-A(4):823-828, 2007.

[34] H. Galperin and A. Wigderson. Succinct representations of graphs. Information and Control, 56:183-198, 1983.

[35] C. Gavoille. A survey on interval routing. Theoretical Computer Science, 245(2):217-253, 2000. 
[36] C. Gavoille and C. S. 0002. Sparse spanners vs. compact routing. In R. Rajaraman and F. M. auf der Heide, editors, Proceedings of the 23rd Annual ACM Symposium on Parallelism in Algorithms and Architectures, pages 225-234, 2011.

[37] C. Gavoille and N. Hanusse. Compact routing tables for graphs of bounded genus. In J. Wiedermann, P. van Emde Boas, and M. Nielsen, editors, 26th International Colloquium on Automata, Languages and Programming, Lecture Notes in Computer Science 1644, pages 351-360. Springer, July 1999.

[38] C. Gavoille and N. Hanusse. On compact encoding of pagenumber $k$ graphs. Discrete Mathematics and Theoretical Computer Science, 10(3):23-34, 2008.

[39] J. R. Gilbert, J. P. Hutchinson, and R. E. Tarjan. A separator theorem for graphs of bounded genus. Journal of Algorithms, 5(3):391-407, 1984.

[40] M. T. Goodrich. Planar separators and parallel polygon triangulation. Jouranl of Computer and System Sciences, 51(3):374-389, 1995.

[41] J. L. Gross. Topological Graph Theory. Wiley, 1987.

[42] R. Grossi and E. Lodi. Simple planar graph partition into three forests. Discrete Applied Mathematics, 84(1-3):121-132, 1998.

[43] P.-N. Guo, T. Takahashi, C.-K. Cheng, and T. Yoshimura. Floorplanning using a tree representation. IEEE Transactions on CAD of Integrated Circuits and Systems, 20(2):281-289, 2001.

[44] S. L. Hakimi. A problem on rectangular floorplans. In Proceedings of 1988 IEEE International Symposium on Circuits and Systems, volume 2, pages 1533-1536, 1988.

[45] X. He. On floor-plan of plane graphs. SIAM Journal on Computing, 28(6):2150-2167, 1999.

[46] X. He, M.-Y. Kao, and H.-I. Lu. Linear-time succinct encodings of planar graphs via canonical orderings. SIAM Journal on Discrete Mathematics, 12(3):317-325, 1999.

[47] X. He, M.-Y. Kao, and H.-I. Lu. A fast general methodology for information-theoretically optimal encodings of graphs. SIAM Journal on Computing, 30(3):838-846, 2000.

[48] M. Isenburg and J. Snoeyink. Spirale Reversi: reverse decoding of the Edgebreaker encoding. Computational Geometry: Theory and Applications, 20(1-2):39-52, 2001.

[49] A. Itai and M. Rodeh. Representation of graphs. Acta Informatica, 17(2):215-219, 1982.

[50] G. Jacobson. Space-efficient static trees and graphs. In Proceedings of the 30th Annual Symposium on Foundations of Computer Science, pages 549-554, Research Triangle Park, North Carolina, 30 Oct.-1 Nov. 1989. IEEE.

[51] Y. Kajitani. Floorplan and placement. In M.-Y. Kao, editor, Encyclopedia of Algorithms, pages 317-320. Springer, 2008.

[52] S. Kannan, M. Naor, and S. Rudich. Implicit representation of graphs. SIAM Journal on Discrete Mathematics, 5:596-603, 1992. 
[53] M. Y. Kao, N. Occhiogrosso, and S. H. Teng. Simple and efficient compression schemes for dense and complement graphs. Journal of Combinatorial Optimization, 2:351-359, 1999.

[54] K.-i. Kawarabayashi, B. Mohar, and B. Reed. A simpler linear time algorithm for embedding graphs into an arbitrary surface and the genus of graphs of bounded tree-width. In Proceedings of the 49th Annual Symposium on Foundations of Computer Science, pages 771-780, 2008.

[55] K. Keeler and J. Westbrook. Short encodings of planar graphs and maps. Discrete Applied Mathematics, 58(3):239-252, 1995.

[56] S. Krivograd, M. Trlep, and B. Zalik. A hexahedral mesh connectivity compression with vertex degrees. Computer-Aided Design, 40(12):1105-1112, 2008.

[57] Y.-T. Lai and S. M. Leinwand. Algorithms for floorplan design via rectangular dualization. IEEE Transactions on CAD of Integrated Circuits and Systems, 7(12):1278-1289, 1988.

[58] C.-H. Li, S. Sonalkar, and L. P. Carloni. Exploiting local logic structures to optimize multicore SoC floorplanning. In Proceedings of Design, Automation and Test in Europe, pages 12911296. IEEE, 2010.

[59] X. Li, Y. Ma, and X. Hong. A novel thermal optimization flow using incremental floorplanning for 3D ICs. In Proceedings of the 14th Asia South Pacific Design Automation Conference, pages 347-352. IEEE, 2009.

[60] Z. Li, X. Hong, Q. Zhou, J. Bian, H. H. Yang, and V. Pitchumani. Efficient thermal-oriented 3D floorplanning and thermal via planning for two-stacked-die integration. ACM Transactions on Design Automation of Electronic Systems, 11(2):325-345, 2006.

[61] Z. Li, X. Hong, Q. Zhou, Y. Cai, J. Bian, H. H. Yang, V. Pitchumani, and C.-K. Cheng. Hierarchical 3-D floorplanning algorithm for wirelength optimization. IEEE Transactions on Circuits and Systems, 53(12):2637-2646, 2006.

[62] J.-M. Lin and Y.-W. Chang. TCG: A transitive closure graph-based representation for general floorplans. IEEE Transactions on VLSI Systems, 13(2):288-292, 2005.

[63] R. J. Lipton and R. E. Tarjan. A separator theorem for planar graphs. SIAM Journal on Applied Mathematics, 36:177-189, 1979.

[64] H. Lopes, G. Tavares, J. Rossignac, A. Szymczak, and A. Safanova. Edgebreaker: a simple compression for surfaces with handles. In Proceedings of the 7th ACM Symposium on Solid Modeling and Applications, pages 289-296, 2002.

[65] H.-I. Lu. Linear-time compression of bounded-genus graphs into information-theoretically optimal number of bits. In Proceedings of the 13th Annual ACM-SIAM Symposium on Discrete Algorithms, pages 223-224, 2002.

[66] H.-I. Lu. Improved compact routing tables for planar networks via orderly spanning trees. SIAM Journal on Discrete Mathematics, 23(4):2079-2092, 2010. 
[67] H.-I. Lu and C.-C. Yeh. Balanced parentheses strike back. ACM Transactions on Algorithms, 4(3):28.1-28.13, 2008.

[68] K. Maling, S. H. Mueller, and W. R. Heller. On finding most optimal rectangular package plans. In Proceedings of the 19th Conference on Design Automation, pages 663-670, 1982.

[69] M. D. Moffitt, J. A. Roy, I. L. Markov, and M. E. Pollack. Constraint-driven floorplan repair. ACM Transactions on Design Automation of Electronic Systems, 13(4):67.1-67.13, 2008.

[70] B. Mohar. A linear time algorithm for embedding graphs in an arbitrary surface. SIAM Journal on Discrete Mathematics, 12(1):6-26, 1999.

[71] J. I. Munro and V. Raman. Succinct representation of balanced parentheses, static trees and planar graphs. In Proceedings of the 38th Annual Symposium on Foundations of Computer Science, pages 118-126, Miami Beach, Florida, 20-22 Oct. 1997. IEEE.

[72] J. I. Munro and V. Raman. Succinct representation of balanced parentheses, static trees and planar graphs. SIAM Journal on Computing, 31(3):762-776, 2001.

[73] J. I. Munro, V. Raman, and A. Storm. Representing dynamic binary trees succinctly. In Proceedings of the 12th Annual ACM-SIAM Symposium on Discrete Algorithms, pages 529-536, 2001.

[74] M. Naor. Succinct representation of general unlabeled graphs. Discrete Applied Mathematics, 28:303-307, 1990.

[75] C. H. Papadimitriou and M. Yannakakis. A note on succinct representations of graphs. Information and Control, 71:181-185, 1986.

[76] M. Patrascu. Succincter. In Proceedings of the 49th Annual IEEE Symposium on Foundations of Computer Science, pages 305-313. IEEE Computer Society, 2008.

[77] D. Peleg. Distributed Computing: A Locality-Sensitive Approach. Monographs on Discrete Mathematics and Applications. SIAM, 2000.

[78] S. Pettie and V. Ramachandran. An optimal minimum spanning tree algorithm. Journal of the ACM, 49(1):16-34, 2002.

[79] D. Poulalhon and G. Schaeffer. Optimal coding and sampling of triangulations. Algorithmica, 46(3-4):505-527, 2006.

[80] J. Rossignac. Edgebreaker: Connectivity compression for triangle meshes. IEEE Transactions on Visualization and Computer Graphics, 5(1):47-61, 1999.

[81] J. Rossignac, A. Safonova, and A. Szymczak. 3D compression made simple: Edgebreaker on a corner-table. In Shape Modeling International Conference, 2001.

[82] J. Rossignac and A. Szymczak. Wrap\&Zip decompression of the connectivity of triangle meshes compressed with Edgebreaker. Computational Geometry: Theory and Applications, 14(1-3):119-135, 1999. 
[83] K. Sadakane and G. Navarro. Fully-functional succinct trees. In Proceedings of the 21st Annual ACM-SIAM Symposium on Discrete Algorithms, pages 134-149, 2010.

[84] D. Saha and S. Sur-Kolay. Encoding of floorplans through deterministic perturbation. In Proceedings of the 22nd International Conference on VLSI Design, pages 315-320. IEEE, 2009.

[85] J. Snoeyink and M. van Kreveld. Linear-time reconstruction Delaunay triangulations with applications. In Proceedings of the 5th Annual European Symposium on Algorithms, Lecture Notes in Computer Science 1284, pages 459-471. Springer, 1997.

[86] S. Sridharan, M. DeBole, G. Sun, Y. Xie, and V. Narayanan. A criticality-driven microarchitectural three dimensional (3D) floorplanner. In Proceedings of the 14th Asia South Pacific Design Automation Conference, pages 763-768. IEEE, 2009.

[87] L. J. Stockmeyer. Optimal orientations of cells in slicing floorplan designs. Information and Control, 57(2-3):91-101, 1983.

[88] T. Suel and J. Yuan. Compressing the graph structure of the web. In Proceedings of the 11th Data Compression Conference, pages 213-222. IEEE, 2001.

[89] A. Szymczak, D. King, and J. Rossignac. An Edgebreaker-based efficient compression scheme for regular meshes. Computational Geometry: Theory and Applications, 20(1-2):53-68, 2001.

[90] T. Takahashi, R. Fujimaki, and Y. Inoue. A ( $4 n-4)$-bit representation of a rectangular drawing or floorplan. In Proceedings of the 15th Annual International Conference on Computing and Combinatorics, pages 47-55, 2009.

[91] K. Tani, S. Tsukiyama, S. Shinoda, and I. Shirakawa. On area-efficient drawings of rectangular duals for VLSI floor-plan. Mathematical Programming, 52(1):29-43, 1991.

[92] G. Taubin and J. Rossignac. Geometric compression through topological surgery. ACM Transactions on Graphics, 17(2):84-115, 1998.

[93] C. Thomassen. The graph genus problem is NP-complete. Journal of Algorithms, 10(4):568$576,1989$.

[94] M. Thorup. Compact oracles for reachability and approximate distances in planar digraphs. Journal of the ACM, 51(6):993-1024, 2004.

[95] M. Thorup and U. Zwick. Compact routing schemes. In Proceedings of the 13th Annual ACM Symposium on Parallel Algorithms and Architecture, pages 1-10. ACM PRESS, 2001.

[96] G. Turán. On the succinct representation of graphs. Discrete Applied Mathematics, 8:289-294, 1984.

[97] W. T. Tutte. A census of planar triangulations. Canadian Journal of Mathematics, 14:21-38, 1962.

[98] R. Wang, E. Young, and C.-K. Cheng. Representing topological structures for 3-D floorplanning. In Proceedings of the International Conference on Communications, Circuits and Systems, pages 1098-1102, 2009. 
[99] R. Wang, E. F. Y. Young, Y. Zhu, F. C. Graham, R. Graham, and C.-K. Cheng. 3-D floorplanning using labeled tree and dual sequences. In Proceedings of the 2008 International Symposium on Physical Design, pages 54-59, New York, NY, USA, 2008. ACM.

[100] S. Wimer, I. Koren, and I. Cederbaum. Floorplans, planar graphs, and layouts. IEEE Transactions on Circuits and Systems, 35(3):267-278, 1988.

[101] D. F. Wong, H. W. Leong, and C. L. Liu. Simulated Annealing for VLSI Design. Kluwer Academic Publishers, 1988.

[102] D. F. Wong and C. L. Liu. Floorplan design of VLSI circuits. Algorithmica, 4(2):263-291, 1989.

[103] K. Yamanaka and S.-I. Nakano. Coding floorplans with fewer bits. IEICE Transactions on Fundamentals of Electronics, Communications and Computer Sciences, E89-A(5):1181-1185, 2006.

[104] K. Yamanaka and S.-I. Nakano. A compact encoding of rectangular drawings with efficient query supports. IEICE Transactions on Fundamentals of Electronics, Communications and Computer Sciences, 91-A(9):2284-2291, 2008.

[105] K. Yamanaka and S.-I. Nakano. A compact encoding of plane triangulations with efficient query supports. Information Processing Letters, 110(18-19):803-809, 2010.

[106] B. Yao, H. Chen, C.-K. Cheng, and R. Graham. Floorplan representations: complexity and connections. ACM Transactions on Design Automation of Electronic Systems, 8(1):55-80, 2003.

[107] K.-H. Yeap and M. Sarrafzadeh. Floor-planning by graph dualization: 2-concave rectilinear modules. SIAM Journal on Computing, 22(3):500-526, 1993.

[108] E. F. Y. Young. Slicing floorplan orientation. In M.-Y. Kao, editor, Encyclopedia of Algorithms, pages 852-855. Springer, 2008.

[109] H. A. Zhao, C. Liu, Y. Kajitani, and K. Sakahushi. EQ-sequences for coding floorplans. IEICE Transactions on Fundamentals of Electronics, Communications and Computer Sciences, 87A(12):3244-3250, 2004.

[110] C. Zhuang, K. Sakanushi, L. Jin, and Y. Kajitani. An extended representation of Q-sequence for optimizing channel-adjacency and routing-cost. In Proceedings of the 2003 Conference on Asia South Pacific Design Automation, pages 21-24, 2003. 